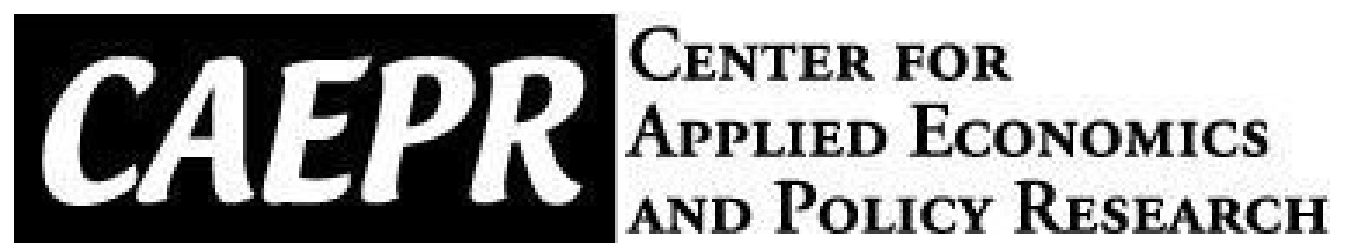

\author{
CAEPR \\ Working Paper \\ \#2020-001
}

\title{
Recursive Utility and Turnpike Theory for \\ GMM Thompson Aggregators
}

\author{
Robert A. Becker \\ Department of \\ Economics \\ Indiana University \\ Juan Pablo Rincón- \\ Zapateroz \\ Departamento de \\ Economia Universidad \\ Carlos III de Madrid
}

January 17, 2020

This paper can be downloaded without charge from the Social Science Research

Network electronic library at https://papers.ssrn.com/sol3/abstract_id=3521498

The Center for Applied Economics and Policy Research resides in the Department of Economics at Indiana University Bloomington. CAEPR can be found on the Internet at: http://www.indiana.edu/ caepr. CAEPR can be reached via email at caepr@indiana.edu or via phone at 812-855-4050.

(C)2019 by Robert A. Becker and Juan Pablo Rincón-Zapatero. All rights reserved. Short sections of text, not to exceed two paragraphs, may be quoted without explicit permission provided that full credit, including () notice, is given to the source. 


\title{
Recursive Utility and Turnpike Theory for GMM Thompson Aggregators*
}

\author{
Robert A. Becker ${ }^{\dagger}$ \\ Department of Economics \\ Indiana University \\ Bloomington, IN 47405 \\ USA
}

\author{
Juan Pablo Rincón-Zapatero \\ Departamento de Economia \\ Universidad Carlos III de Madrid \\ 28903 Getafe (Madrid) \\ Spain
}

January 17, 2020

\begin{abstract}
The existence of a unique optimum, a unique optimal stationary program, and a turnpike theorem are demonstrated for a neoclassical one sector optimal growth model. The planner's allocation problem is formulated as a discrete time deterministic, infinite horizon programming model. The production sector is subject to diminishing marginal returns to capital. The planner's objective function is derived from a Generalized Marinacci and Montrucchio (GMM) Thompson aggregator preference. A given Thompson aggregator may be associated with many intertemporal utility functions (which may not be ordinally equivalent). The choice of one of these representations over another is shown to be a matter of mathematical tractability. There is an observational equivalence between those alternative objective functions: the qualitative features of the optimal solution do not depend on the particular utility function representation of the underlying Thompson aggregator preference structure.
\end{abstract}

JEL Codes: D15, D50,E10, E13

Keywords: Recursive Utility, Thompson Aggregators, Generalized MarinacciMontrucchio Aggregators, Koopmans Equation, Extremal Fixed Points, Turnpike Theorem, Discounted Golden-Rule, Observational Equivalence Theorem

\footnotetext{
*Our papers [4], [5], and Marinacci and Montrucchio's [19] contain background material on Thompson aggregators not included in the present article. We have benefited from comments on a preliminary version by participants at the conference on "Real and Financial Interdependencies: Instablity, International Openness and Regulation Policies," hosted by the Aix-Marseille School of Economics, June 24-26, 2019. We thank in particular Christian Ghiglino and our discussant, Mauro Bambi, for sharing their insights and comments that improved the paper. Juan Pablo Rincón-Zapatero acknowledges research support from the Ministerio de Ciencia, Innovación y Universidades - Agencia Estatal de Investagación, Grant ECO2017-86261-P.

†becker@iu.edu or becker@indiana.edu

$\ddagger$ jrincon@eco.uc3m.es
} 


\section{Introduction}

The development of recursive utility functions from aggregators is now wellestablished following the seminal work by Lucas and Stokey [17]. Boyd [10] extended their work to include optimal growth models with possibly indefinitely growing capital accumulation. Becker and Boyd [3] expanded on those papers as well as the fundamental studies by Beals and Koopmans [6] and Magill and Nishimura [18] on optimal growth with aggregator preferences in one-sector models. Becker and Boyd's [3] book covered how the Blackwell class of aggregators expressed intertemporal utility functions and yielded turnpike theorems in a variety of one sector optimal growth models.

We note that those turnpike results do not require all the defining conditions of Blackwell aggregators. We find that qualitative results on optimal accumulation of capital and consumption may be demonstrated when aggregator preferences are not members of the Blackwell family. Neither Beals and Koopmans [6] nor Magill and Nishimura [18] gave explicit functional form examples outside the discounted time additive utility framework. ${ }^{1}$ Indeed, neither paper addresses the recovery of intermporal utility for the underlying infinite horizon models given a specified aggregator preference primitive. They placed assumptions directly on the aggregator sufficient for monotone optimal capital sequences and their convergence to some long-run steady state capital stock. We build on this flexible structure to expand the available results from the Blackwell family to apply as well to some Thompson aggregators. Our turnpike theory applies to a collection of aggregators we call the Generalized Marinacci-Montrucchio [19] (GMM) aggregator preferences. This class includes one of their original examples as well as the CES examples studied by us [4].

Aggregators are functions of two variables - current consumption and (discounted) future utility. The Blackwell aggregator class builds in discounting of future utilities by means of a global Lipschitz condition imposed on the aggregator's second argument. The Lipschitz constant must be smaller than one. The newer class of Thompson aggregators introduced by Marinacci and Montrucchio [19] violate this Lipschitz condition. The Thompson aggregators studied in our paper fail to satisfy the Blackwell Lipschitz condition on their entire domain. However, they are locally Lipschitz on open subsets of their domain. The Lipschitz constant may vary from one open set to another. ${ }^{2}$ We will see that the Lipschitz constant can take a value less than one at particular combinations of current consumption and future utility values. Indeed, this turns out to be the case at both the model's Golden-Rule consumption-utility pairing as well as in the case of the unique stationary optimal growth solution developed in Sections 5 and 6.

The purpose of our paper is to demonstrate a turnpike theorem is available

\footnotetext{
${ }^{1}$ Beals and Koopmans ([6], pp. 1013-1014) characterize some aggregator examples consistent with the Blackwell restrictions developed later. Their only explicit functional form example yields a time additive discounted utility. Koopmans, et al [16], gives the first example of a nonadditive aggregator preference.

${ }^{2}$ A locally Lipschitz function is also known as a Lipschitz continuous function here.
} 
for one-sector technology models subject to diminishing marginal returns on capital when Thompson aggregator preferences are specified by members of the GMM aggregator class introduced below. Our work draws on the basic optimal growth turnpike characterization results due to Beals and Koopmans [6] and Magill and Nishimura [18] as synthesized by Becker and Boyd [3]. Qualitative properties of optimal capital sequences such as non-crossing, monotonicity and convergence to a long-run steady state capital stock are demonstrated using assumed properties of aggregators that do not impose membership in the Blackwell class. The approach taken in those works is shown here to be robust in the sense that GMM Thompson aggregators satisfy their sufficient conditions for a turnpike theorem. Previous applications of their results for recursive, but not additive utility, focused on aggregators contained in the Blackwell class (see Becker and Boyd [3] for details). Given the subsequent interest in the Thompson aggregators since Marinacci and Montrucchio's publication [19], it is important to establish Thompson aggregators are an applicable criterion in optimal growth theory. We affirm this possibility for the class of GMM Thompson aggregators.

We demonstrate the Turnpike Theorem for a particular choice of an intertemporal utility representation among the many possible utility functions that represent the GMM aggregator preference. This choice is necessary as a starting point in our optimal growth model. There can be several solutions to the Koopmans equation (see Section 2) for a given GMM aggregator. These solutions may NOT be ordinally equivalent! However, they do agree on the interior of the underlying commodity space. This agreement turns out to have an interesting economic consequence: once we have characterized the optimum for our original selection of the planner's objective, the same optimal consumption and capital sequences for that objective also solve the optimum growth problem with any other choice of a utility function satisfying the GMM aggregator's Koopmans equation. This is the observational equivalence property: the planner's observable behavior (its optimal choice of consumption and capital sequences from the same initial capital stock) is independent of the chosen utility function selected from the Koopmans equation's solution set. This result exploits all the information available to us from the existence of an optimum to the Turnpike Theorem as well as foundational results on the existence and uniquenss of recursive utility functions derived from Thompson aggregators (including the GMM cases).

Writing from a methodological perspective, Koopmans [14] proposed that researchers use optimal growth problems as mathematical screening devices for finding the possibly hidden implications of welfare judgments implicit in the planner's utility function. A screening exercise would find the consequences of those judgments by examining the model's optimal solution and its qualitative (or, even quantitative) features. Our observational equivalence result tells us that the choice of a particular utility consistent with the GMM aggregator preference does NOT matter from this mathematical screening perspective as each objective has the same optimum. So, even though we cannot say that one representation is ordinally equivalent to another, we can infer that optimal behavior is the same no matter which representation is postulated as the planner's objective given the technological constraints and endowments remain the same. 
This is the subject of Section 7. Concluding comments are presented in Section 8. Some technical arguments are relegated to appendices.

\section{GMM Thompson Aggregator Preferences}

Thompson aggregators were introduced by Marinacci and Montrucchio [19]. These aggregators satisfy a number of properties detailed in their paper. We bypass their axiomatic formalisms to focus attention on a family of parameterized examples we call the Generalized Marinacci-Montrucchio (GMM) Aggregators. A GMM aggregator preference has the functional form:

$$
W(x, y)=\beta_{1} x^{\eta}+\beta_{2} y^{\rho},
$$

where $(x, y) \geq(0,0)$ and the parameters satisfy:

$$
\begin{aligned}
& 0<\beta_{1} \leq 1 \\
& 0<\beta_{2}, \eta, \rho<1 .
\end{aligned}
$$

The variable $x$ denotes current consumption and $y$ denotes the value of future (discounted) utility.

IF $\eta=\rho$ the GMM aggregator has a constant elasticity of substitution, $\sigma=1 /(1-\rho)>1$. Otherwise, the GMM family may contain aggregators which do not have a constant elasticity of substitution. Indeed, if $\eta \neq \rho$ the aggregator is not homogeneous in $(x, y)$; its elasticity of substitution cannot be a constant.

The particular specification $W(x, y)=\beta_{1} x^{\eta}+\beta_{2} \sqrt{y}$ appears in Section 5.3 where it plays an important role our detailed steady state theory. The utility values derived from this aggregator evaluated for a constant consumption sequence solve a particular functional equation. Two explicit solutions to that equation are calculated in Appendix 3. Those solutions, when the parameter values are $\eta=1 / 2$ and $\beta_{1}=1$, form the basis for the figures displayed in Section 5.3. This aggregator example's constant elasticity of substitution is two.

We highlight two members of the GMM class. The first yields the MM Thompson aggregators specified by the formula:

$$
W(x, y)=x^{\eta}+\beta y^{\rho},
$$

where $(x, y) \geq(0,0)$ and $0<\eta, \beta, \rho<1$. Here, $\beta_{1}=1$ and $\beta_{2} \equiv \beta$. The MM aggregator aggregator has a variable elasticity of substitution when $\eta \neq \rho$. This family was originally given as an example by Marinacci and Montrucchio [19] and, suitably generalized here, yields the GMM aggregator preferences.

The second class consists of the Symmetric CES Thompson aggregators:

$$
W(x, y)=\beta x^{\rho}+(1-\beta) y^{\rho},
$$

where $(x, y) \geq(0,0), 0<\beta<1$, and $0<\rho<1$. Clearly $\sigma>1$ with this specification. Unlike classical demand theory the aggregator $W$ and the aggregator $V=W^{1 / \rho}$ do not represent the same underlying preference relation on 
the commodity space. ${ }^{3}$ Hence, the elasticity of substitution identified in $W$ is not an ordinal property of the underlying aggregator preference relation. We usually drop the term Symmetric and refer to the CES class more generally.

Given a GMM aggregator, the Inada Conditions in $W_{2}(x, y)$ are, for a given $x$, the properties: $W_{2}(x, y) \rightarrow+\infty$ as $y \rightarrow 0^{+}$and $W_{2}(x, y) \rightarrow 0$ when $y \rightarrow \infty$, where $W_{2}$ is the partial derivative of $W$ with respect to $y$; interpret $W_{1}$ similarly. Each GMM aggregator is additive separable between $x$ and $y$ with $W_{2}(x, y)=\left(\rho \beta_{2}\right) / y^{1-\rho}$. The GMM $W$, satisfies the Inada conditions and fails to satisfy a global Lipschitz condition in its second argument. This implies $W$ cannot be a member of the Blackwell aggregator class. However, $W$ is strictly concave in its second argument for a fixed value of $x$, hence it is Lipschitz continuous on each open set in the $y$-variable's domain. The GMM aggregators are concave Thompson aggregators since $\mathrm{W}$ is a concave function of $(x, y)$ with our parametric assumptions. In fact, they are increasing and strictly concave functions in $(x, y)$.

The GMM aggregators have a fixed point property corresponding to the solution(s), $y(x)$, of the functional equation $W(x, y(x))=y(x)$. This equation has a solution in the class of bounded functions defined on $[0, b]$, where $b>0$ is the maximum sustainable capital stock given the productive technology. At $x=$ 0 , an example (Section 5.3 and Appendix 3 ) shows there may be two solutions: one has $y(0)=0$ and the other $y(0)>0 .{ }^{4}$ Marinacci and Montrucchio's [19] axiomatic requirements for a Thompson aggregator requires $W(x, y)=y$ has at least one nonnegative solution for each $x \geq 0$. We refine this in the GMM class as Inada conditions hold in $W_{2}(x, y)$ for a given $x>0$. Notice that $W$ satisfies a local Lipschitz condition at $y=y(x)$ whenever $x>0$. This is a also consequence of strict concavity of $W$ in $y$ for each $x>0$. This local Lipschitz property turns out to be critical in our proof that a stationary optimal program exists (Proposition 3).

Intertemporal preferences are formally defined in terms of a utility function, denoted $U$, over sequences of consumption, $C=\left\{c_{t}\right\}_{t=1}^{\infty}$, with $c_{t} \geq 0$ for each $t=1,2, \ldots$. We write $C=\left\{c_{t}\right\}$ and it is understood the sequence is indexed by the natural number $t$. Stationary consumption sequences are denoted $C_{c o n}=\{c\}$ where $c_{t}=c \geq 0$ for each $t$. Utility may also be defined for $\theta_{\text {con }}=\{0\}$, the zero sequence. Similarly capital sequences are denoted by $K=\left\{k_{t-1}\right\}$ where $k_{0}=k$ is the given initial capital stock. Here, con-

\footnotetext{
${ }^{3}$ Koopmans [13] defines ordinally equivalent aggregators that are meant to correspond to ordinally equivalent utility functions. $V$ is Koopmans equivalent to $W$ provided there is a strictly increasing transformation $\Phi$ such that $V\left(x, y^{*}\right)=\Phi\left(W\left(x, \Phi^{-1}(y)^{*}\right)\right.$. Here, the transformation only changes the scale of utility values and $y^{*}$ is the new utility scale obtained by applying $\Phi$ to the original utility scale, $y$. The economic point is that the recursive utility function is self-referential when expressed via an aggregator and that means the $y^{*}$ - value in $V$ must also be transformed inside $W$ to put it in terms of the $y$-scale.

${ }^{4}$ See Appendix 3 for an analysis of this functional equation. We construct an example where there are two solutions: a smallest and a largest. The former is a lower-semicontinuous and concave function on its domain. The latter is, in general, upper-semicontinuous on its domain. However, it is continuous in our example. Both solutions agree on $(0, b]$. Hence, $y(x)$ is a continuous function on $(0, b]$. The lower solution has a downward jump discontinuity at 0 .
} 
sumption (and capital) sequences are elements of the nonnegative cone of the space of all bounded real-valued sequences, denoted $\ell_{\infty}^{+}$with its usual sup norm topology unless otherwise noted. This positive cone has a nonempty norm interior, which we denote by $\ell_{\infty}^{++}$. The corresponding boundary of the positive cone is $\partial \ell_{\infty} \equiv \ell_{\infty}^{+} \backslash \ell_{\infty}^{++}$. The aggregator approach recovers this utility function (up to ordinal equivalence) from a prespecified aggregator taken as the model's primitive expression of intertemporal consumption preferences. The recovery and uniqueness theories in Marinacci and Montrucchio ([19], [20]), our working papers ([4],[5]), and Balbus [1] prove each GMM aggregator preference yields a unique utility representation for consumption sequences in $\ell_{\infty}^{+}$satisfying $\liminf \operatorname{in}_{t \rightarrow \infty} c_{t}>0$. This latter restriction implies that the recovered utility function, $U$, is uniquely determined by the given aggregator preference on $\ell_{\infty}^{++} .5$

The planner's objective function is a recursive utility function, $U$, derived from a given GMM aggregator, that satisfies the recursive relationship (the Koopmans equation):

$$
U(C)=W\left(c_{1}, U(S C)\right)
$$

where $S C=\left(c_{2}, c_{3}, \ldots\right)$ denotes the shift operator. General recovery theory deriving utility representations of the given aggregator preference may yield multiple solutions of the Koopmans equation. The problem of demonstrating the existence of solutions to this functional equation is formally equivalent to solving a nonlinear operator problem whose fixed points are the solutions. This approach finds representations of the planner's preferences from a given GMM aggregator as fixed points of the Koopmans operator, denoted by $T_{W}$. The operator is a monotone self-map on an order interval of bounded functions (details in Appendix 1). Each fixed point of this operator solves the Koopmans equation and determines a particular utility representation of the aggregator. In particular, there are extremal fixed points - a Least and Greatest Fixed Point (LFP and GFP, respectively). We focus on these solutions as they are "constructed" by iteration of the Koopmans operator over the natural numbers (each from a different initial seed). Marinacci and Montrucchio [19] single out the LFP as the preferred solution - it corresponds to what Kantorovich [12] refers to as the operator problem's principal solution. ${ }^{6}$

The GFP of the Koopmans operator is $U^{\infty}$, where $U^{\infty}=T_{W} U^{\infty}$ and $U \leq$ $U^{\infty}$ whenever $U=T_{W} U$. The LFP is $U_{\infty}$ and $U_{\infty} \leq U$ for each fixed point, $U$. Appendix 1 sketches the Recovery Theory for the special case of our optimal growth problem where the utility and aggregator domains are restricted by technological considerations as developed below. Both extremal fixed points

\footnotetext{
${ }^{5}$ We show in our working papers the Koopmans equation can admit multiple solutions which fail to agree at consumption sequences satisfying $\lim \inf _{t \rightarrow \infty} c_{t}=0$. . Nonuniqueness examples may also be found in Bloise and Vialikis [9]. The GMM example deployed in Section 5.3 and Appendix 3 also fails uniqueness of its utility representations at the zero constant consumption sequence.

${ }^{6}$ Becker and Rincón-Zapatero [4] present extensive arguments why it is reasonable to think of the LFP as the problem's principal solution. There is no reason to believe these extremal solutions are ordinally equivalent to one another. That is, neither may be Koopmans equivalent to the other.
} 
are concave functions and increasing in each argument separately. The LFP is lower-semicontinuous on its domain with the product topology, and satisfies $U_{\infty}\left(\theta_{\text {con }}\right)=0$. The GFP is upper-semicontinuous in the product topology and shown to be a strictly concave function in Appendix 2. We note that $U^{\infty}\left(\theta_{\text {con }}\right) \geq U_{\infty}\left(\theta_{\text {con }}\right)=0$ and a strict inequality may hold as well.

\section{Optimal Growth Problems}

We propose a family of optimum growth problems parameterized by the utility function representation of $W$ and the initial capital stock's magnitude. Let fix $\left(T_{W}\right)$ be Koopmans operator's set of fixed points (which is nonempty). Each $U \in \operatorname{fix}\left(T_{W}\right)$ is a bounded and nonnegative real-valued function on the restricted domain $[0, b]^{\infty} \subset \ell_{\infty}^{+}$, where $b>0$, and $[0, b]^{\infty}$ is the countable Cartesian copy of the intervals $[0, b]$. It is understood $U$ is a utility derived from a GMM aggregator preference.

Let $U \in \operatorname{fix}\left(T_{W}\right)$. Then $U_{\infty} \leq U \leq U^{\infty}$. We formulate the Ramsey planner's optimal growth problem for each $U \in$ fix $\left(T_{W}\right)$ as follows:

$$
\sup U(C)
$$

by choices of $C=\left\{c_{t}\right\}, K=\left\{k_{t-1}\right\}$ subject to

$$
\begin{aligned}
c_{t}+k_{t} & \leq f\left(k_{t-1}\right),(t=1,2, \ldots) ; \\
c_{t} & \geq 0, k_{t} \geq 0 \quad(\text { each } t) ; \\
k_{0} & =k>0 \text { given } .
\end{aligned}
$$

Here $f$ is the standard one-sector production function with fixed labor input and variable capital input. Impose the standard conditions: $f(0)=0, f^{\prime}>0, f^{\prime \prime}<$ $0, f^{\prime}(0+)=+\infty$, and $f^{\prime}(k)<1$ whenever $k>b=f(b)>0$. The capital input $b$ is the maximum sustainable stock. It serves to restrict the domains of the given GMM aggregator and its utility representations in fix $\left(T_{W}\right)$. The wellknown Cobb-Douglas production function $f(k)=A k^{\alpha}$ with parameters $A>0$ and $0<\alpha<1$ satisfies these conditions. We formally refer to this optimal growth problem by $\mathcal{P}_{U}(k)$ to signify the problem's dependence on the choice of utility representation in fix $\left(T_{W}\right)$ and the magnitude of the initial capital stock, $k$. We suppress the explicit dependence of the production function in this notation for the optimum growth problem. We use the notation $\mathcal{P}_{\infty}(k) \equiv \mathcal{P}_{U_{\infty}}(k)$ and $\mathcal{P}^{\infty}(k) \equiv \mathcal{P}_{U^{\infty}}(k)$ for the optimal growth models specified by the LFP and GFP, respectively.

Consumption and capital sequences ( $C$ and $K$, respectively) satisfying the constraints are said to be feasible. The set of feasible consumption programs is $F(k)$. If $C \in F(k)$, then there is a corresponding sequence $K$ such that $C$ and $K$ are feasible. The set $F(k)$ is nonempty for each $k$, with $0 \leq k \leq b$. The conditions on the technology imposed as restrictions of the production function imply that feasible sequences are elements of $\ell_{\infty}^{+}$. In fact, for each feasible consumption and capital sequence, $0 \leq c_{t}, k_{t-1} \leq b$ holds for each $t$ given 
$0 \leq k \leq b$. The technology conditions applied to the production function and initial endowment of capital imply $F(k) \subset[0, b]^{\infty}$ and $F(k)$ is a nonempty, compact, convex subset of $\ell_{\infty}^{+}$with its (relative) product topology. We now formally restrict the domains of the utility functions to $[0, b]^{\infty} \subset \ell_{\infty}^{+}$whenever that utility satisfies the Koopmans equation. Utility values are also uniformly bounded in the sense that each $U \in \operatorname{fix}\left(T_{W}\right)$ is bounded above by the number $y_{b}$, which depends on $b .^{7}$ Appendix 1 presents further detail on this boundedness property.

If $K \in[0, b]^{\infty}$, the constraints uniquely determine a corresponding consumption sequence according to the formula $c_{t}=f\left(k_{t-1}\right)-k_{t}$, with $k_{0}=k$. Clearly $C$ and $K$ are feasible sequences then whenever $f\left(k_{t-1}\right)-k_{t} \geq 0$ for each $t$ $(t=1,2 \ldots)$. For this reason the Turnpike Theorem characterizes the optimal capital sequence. The analogous properties exhibited for the corresponding consumption sequence are readily deduced. The Existence and Uniqueness Theorem operate directly with the feasible consumption sequences. The optimal capital sequences are determined from the optimum consumption sequence by the feasibility conditions with $k_{0}=k$.

\section{Existence and Uniqueness of the Optimal So- lution to $\mathcal{P}^{\infty}(k)$}

We assign the planner the objective function, $U^{\infty} \in \operatorname{fix}\left(T_{W}\right)$. It is uppersemicontinuous in the product topology on $[0, b]^{\infty}$ viewed as a subspace of $\ell_{\infty}^{+}$ endowed with the product topology. ${ }^{8}$ It is also increasing in each argument and strictly concave on $[0, b]^{\infty}$. The formal proof that $U^{\infty}$ is strictly concave is given in Appendix 2. We verify the optimum's existence by a direct method (e.g. via the well-known Weierstrass Theorem) since the product topology where $U^{\infty}$ is upper-semincontinuous is also the same topology for which $F(k)$ is compact. As the GFP is a strictly concave function and $F(k)$ is convex, we also deduce $\mathcal{P}^{\infty}(k)$ has a unique solution.

This direct approach is not available for any the $U \in \operatorname{fix}\left(T_{W}\right)$. For example, the difficulty with the use of the LFP is that it is only product lowersemicontinuous on $F(k)$ and may not be upper-semicontinuous. ${ }^{9}$ We employ an indirect method to prove the existence of a unique solution to the problem $\mathcal{P}_{\infty}(k)$ where $U_{\infty}$ specifies the planner's objective. That method utilizes the turbpike properties exhibited by the solution to $\mathcal{P}^{\infty}(k)$. We show there is

\footnotetext{
${ }^{7}$ Both Beals and Koopmans [6] and Magill and Nishimura [18] assume that the utility function's values are elements of the unit interval. We are simply taking the requirement that utility values belong to the interval $\left[0, y_{b}\right]$ instead.

${ }^{8} \mathrm{It}$ is also product upper-semicontinuous on norm bounded subsets of $\ell_{\infty}^{++}$according to Marinacci and Montrucchio's Theorem 4 ([19], p. 1790).

${ }^{9}$ Semicontinuity properties of the extremal fixed points are deduced by use of the iterative procedures that yield them in a limiting process. If there are nonextremal fixed points, we cannot directly say anything more general than they are bounded functions! However, the uniqueness theorems for Thompson aggregators imply these noncontstructively obtained fixed points share many properties of the extremal solutions on the commodity space's norm interior.
} 
also a unique solution to $\mathcal{P}_{\infty}(k)$ and this solution agrees with the one found for the GFP! In fact, this holds for ANY choice of $U \in \operatorname{fix}\left(T_{W}\right)$ - a result we call the observational equivalence of the optimum growth path for each choice of the planner's utility in fix $\left(T_{W}\right)$. This is an important result. We do not know if the elements in $\operatorname{fix}\left(\mathrm{T}_{W}\right)$ are ordinally equivalent in the sense of Koopmans [13]. The main issue is these utility functions may differ on $[0, b]^{\infty} \cap \partial \ell_{\infty}$ where consumption sequences converge to zero or have lower limits which converge to zero. However, we prove that the "observable" consequences for optimal growth are the same for any $U \in \operatorname{fix}\left(T_{W}\right)$. The planner's solution does not change when, for example, the LFP replaces the GFP. The GFP optimum problem has a an analytical advantage over the choice of the LFP objective because the GFP's continuity properties agree with our compactness condition on $F(k)$ to yield existence and uniqueness for $\mathcal{P}^{\infty}(k)$. The LFP, viewed as the principal solution of the Koopmans equation, has a potential computational advantage over the GFP. The LFP's interpretation links to the sequence of finite horizon approximations of the planner's problem. Just consider the finite horizon approximations of the LFP from below: $T_{W}^{N} \theta(C)=W\left(c_{1}, W\left(c_{2}, \ldots W\left(c_{N}, 0\right)\right)\right) \nearrow U_{\infty}(C)$, as $N \rightarrow \infty$. Thus, it is important to show a change in the GFP objective to the LFP objective does NOT alter the planner's optimizing behavior - the same optimal capital and consumption sequences emerge. The formal Observational Equivalence Theorem is presented in Section 7 following the Turnpike Theorem.

Let $J^{\infty}(k)=\sup _{C}\left\{U^{\infty}(C): C \in F(k)\right\}$. This optimization problem has a solution, $C^{\infty} \in F(k)$ provided $J^{\infty}(k)=U^{\infty}\left(C^{\infty}\right)$ and we write

$$
C^{\infty} \in \arg \max \left\{C \in F(k): J^{\infty}(k)=U^{\infty}(k)\right\} .
$$

Theorem 1 (Existence and Uniqueness). The optimal growth model $\mathcal{P}^{\infty}(k)$ has a unique optimal solution for each $k, 0 \leq k \leq b$.

Proof. Apply Weierstrass's Theorem: $U^{\infty}$ is product upper-semicontinuous on $[0, b]^{\infty}$ and $F(k)$ is a product compact subset of $[0, b]^{\infty}$. Uniqueness follows since $U^{\infty}$ is a strictly concave function on the convex set $F(k)$ (see Appendix 2).

We let $C(k)=\left\{c_{t}(k)\right\}$ denote this uniquely determined optimal consumption sequence given the parameter $k_{0}=k$. The corresponding capital stock sequence, $K(k)=\left\{k_{t-1}(k)\right\}$, is also uniquely determined at each $t$ given the initial stock and the optimal consumption sequence, $C(k)$. IF $k_{0}=0$, then the technology conditions imply that only zero consumption and the zero capital sequences are feasible, and hence optimal as well. We are interested in non-trivial solutions of the optimum problem where the initial stocks, $k$, are positive. Our turnpike theory explores the properties of non-trivial optimal growth paths for different positive initial endowments of the capital stock. That is, we seek to verify the possibility of interesting economic dynamics as a characteristic of optimal growth with Thompson aggregator preferences. 


\section{Turnpike Theory}

Turnpike theory draws qualitative conclusions about the long-run behavior of the optimal capital sequence. For example, the optimal capital sequence is monotonic and convergent to a long-run stock that does not depend on the choice of the positive initial capital stock. This result is known as the Turnpike Theorem in aggregative capital accumulation models. We show this result obtains in our class of optimal growth problems. The GFP $U^{\infty}$ is taken as the planner's objective function in our optimal growth theory application.

We assume that the optimal capital and consumption sequences are regular in the sense that $0<k_{t-1}(k)<b$ and $c_{t}(k)>0$ for each $t$. That is, $C(k)$ and $K(k)$ are elements of $(0, b)^{\infty}$. Our Turnpike Theorem is derived for regular paths of accumulation and consumption. This "interiority" property is reasonable given that Inada conditions on production hold as well for the aggregator functions in each variable separately. In fact, we verify the regularity of the optimum holds given a positive initial capital stock. The Turnpike Theorem also turns out to imply that the $\inf _{t} c_{t}(k)>0$, so $C(k) \in \ell_{\infty}^{++}$. This turns out to be a critical property for linking the solution of $\mathcal{P}^{\infty}(k)$ to the solution of $\mathcal{P}_{\infty}(k)$.

\subsection{The Turnpike Theorem}

Our main result is recorded below and characterizes the optimal capital sequence given the positive initial capital stock. We maintain the assumptions governing the Existence and Uniqueness Theorem along with the requirement $0<k<b$. The Golden-Rule capital stock and corresponding consumption play an important role in our turnpike theory.

A pair $(c, k) \geq(0,0)$ is stationary if $c=h(k) \equiv f(k)-k \geq 0$. A stationary path is feasible for the optimal growth problem whenever $k_{0}=k$. The optimal stationary program $\left(c^{+}, k^{+}\right)$is defined by

$$
\begin{aligned}
k^{+} & =\arg \max \{h(k): k \in[0, b]\} ; \\
c^{+} & =h\left(k^{+}\right) .
\end{aligned}
$$

The optimal stationary program is unique as $h$ is continuous on $[0, b], h(0)=$ $0=h(b)$, and strictly concave in $k$. The optimal stationary pair is also known as the Golden-rule capital stock, $k^{+}$, and consumption, $c^{+}$. The Golden-Rule pair depends only on the production function and stationarity requirement. It is independent of the choice of an underlying aggregator preference. It is wellknown that $f^{\prime}\left(k^{+}\right)=1$ with $k^{+}, c^{+}>0$ completely characterizes the Golden Rule.

If the initial capital stock $k \in\left(k^{+}, b\right)$, then the stationary pair $(c, k), c=$ $h(k)$ is feasible and well-known to be inefficient (by the Phelps-Koopmans Theorem). Hence, this feasible program cannot be an optimal solution to the Ramsey problem. In particular, such an initial capital stock cannot correspond to a steady state solution for the planner's problem. We show below there is a 
unique stationary pair which is also an optimal program and the steady state stock is strictly less than the Golden-Rule stock. This steady state pair forms the model's “discounted Golden-Rule." Establishment of the existence and uniqueness of the discounted Golden-Rule is a critical step in the proof of our main result.

Theorem 2 Suppose $K(k)=\left\{k_{t-1}(k)\right\}$ is the optimal capital sequence and it is regular for problem $\mathcal{P}^{\infty}(k)$. Then, for each initial capital stock $k, 0<k<b$ :

(I) $\left\{k_{t-1}(k)\right\}$ is strictly increasing in $k$;

(II) $\left\{k_{t-1}(k)\right\}$ is a monotonic sequence for each given $k$;

(III) There exists a unique $k^{*}, 0<k^{*}<k^{+}$, such that

(a) if $k=k^{*}$, then $k_{t}\left(k^{*}\right)=k^{*}$ for each $t(t=1,2, \ldots)$;

(b) if $k \in\left(0, k^{*}\right)$, then $\left\{k_{t-1}(k)\right\} \nearrow k^{*}$;

(c) if $k \in\left(k^{*}, b\right)$, then $\left\{k_{t-1}(k)\right\} \searrow k^{*}$.

The symbols $\nearrow(\searrow)$ to indicate the sequence of capital stocks is strictly increasing (decreasing) and converges to the stationary capital stock, $k^{*}$.

The sufficient condition for (IIIa) and (IIIb), first developed by Beals and Koopmans [6] and elaborated on further by Magill and Nishimura [18], is

$$
\frac{\partial}{\partial x}\left(\frac{W_{1}(x, y)}{W_{2}(x, y)}\right)<0 .
$$

This is the Beals - Koopmans Condition. It implies the marginal rate of impatience (see below) is decreasing in the first period's consumption, $x$, holding future utility value, $y$, constant. This is a behavioral assumption about the planner's time preference for consumption today as against future utility streams. This property is also consistent with a diminishing marginal rate of substitution along level curves of $W$, which follows from the assumed concavity of $W$.

Property $(I)$ is known as the non-crossing property (see Becker and Boyd ([3], p. 162 and p.168). It formally means that if $k<k^{\prime}$ are two initial capital stocks, then $k_{1}(k)<k_{1}\left(k^{\prime}\right)$. Likewise, treating $k_{1}(k)$ and $k_{1}\left(k^{\prime}\right)$ as new initial conditions at time one yields $k_{2}(k)<k_{2}\left(k^{\prime}\right)$, and so on.

Property $(I I)$ is the capital monotonicity property. This property implies the optimal capital sequence has a limit. Property $(I I I)$ tells us the optimal capital sequence converges monotonically to the unique stationary optimal program, denoted $k^{*} \in\left(0, k^{+}\right)$. This is the turnpike property - an asymptotic or long-run statement about the evolution of the economy's capital stock. The optimal capital stock is arbitrarily close to the long-run stationary capital stock, $k^{*}$, for all but a finite number of consecutive periods (and that number depends the desired accuracy of the approximation of the optimal capital sequence by the stationary capital stock, $\left.k^{*}\right)$. 
The Turnpike Theorem yields a nice interpretation of the model's (shadow) interest factor and the corresponding wage rate. ${ }^{10}$ Given the optimal capital sequence, $K(k)$, the interest factor sequence is $1+r_{t}=f^{\prime}\left(k_{t-1}\right)$ and $w_{t}=$ $f\left(k_{t-1}\right)-f^{\prime}\left(k_{t-1}\right) k_{t-1}$ at each time. If $0<k<k^{*}$, then the imputed interest factor is decreasing and the wage rate is increasing with time's passage. The optimal path is consistent with the Orthodox Vision of Capital Theory as described by Bliss [8]. Interest rates fall and wage income rises as capital accumulates.

There are two steps in the proof. First, we show GMM aggregator preferences satisfy the Beals - Koopmans condition for the Monotonicity and Recursive Turnpike Theorems as exposited in Becker and Boyd ([3], pp. $159-162 ; 167-170)$. Verification that (5) holds for each GMM aggregator is an easy computation. The second step is to prove the existence and uniqueness of the stationary optimal program, or steady state, for the given optimal growth problem. This is essential since we want to know that in the long-run optimal accumulation programs approach THE stationary optimal capital stock.

Properties of the optimal consumption sequence follow from the aforementioned theorem. Let $c^{*}=f\left(k^{*}\right)-k^{*}>0$. Then $\left\{c_{t}(k)\right\} \nearrow(\searrow) c^{*}$ according to $k<(>) k^{*}$.

\subsection{Impatience Concepts and Optimality Conditions}

Define the partial derivative, $U_{t}^{\infty}(C)=\partial U^{\infty} / \partial c_{t}$ for any $C=\left\{c_{t}\right\} \in[0, b]^{\infty}$, provided $c_{t}>0$. These partial derivatives exist when $W$ is a GMM aggregator (it is at least twice continuously differentiable on the interior of $\mathbb{R}_{+}^{2}$ ). An easy calculation shows

$$
U_{t}^{\infty}(C)=W_{2}\left(c_{1}, U^{\infty}(S C)\right) \cdots W_{2}\left(c_{t-1}, U^{\infty}\left(S^{t-1} C\right)\right) \cdot W_{1}\left(c_{t}, U^{\infty}\left(S^{t} C\right)\right),
$$

where the shift operator $S^{t}=S\left(S^{t-1}\right)$, for $t=1,2, \ldots$, and $S^{0}$ is the identity $S^{0} C=C$. Clearly, $S^{t-1} C=\left\{c_{t}, c_{t+1}, \ldots\right\}$, etc. If we fix all components of $C$, except at time index $t$, and let $c_{t} \rightarrow 0, c_{t}>0$, we see $U_{t}^{\infty}(C) \rightarrow \infty$ since $W_{1}\left(c_{t}, U^{\infty}\left(S^{t} C\right)\right) \rightarrow \infty$ (and note that $S^{t} C$ does not depend on $c_{t}$ ). This Inada condition implies that optimal consumption at any time is positive provided the initial capital stock is positive (and, hence, the corresponding capital stock is positive): the optimal consumption and capital sequences are regular.

The marginal rate of impatience, $R(\cdot)$, is defined at each time $t$, for a given consumption sequence $C$, by the lefthand side of the equation:

$$
1+R\left(S^{t-1} C\right)=\frac{W_{1}\left(c_{t}, U^{\infty}\left(S^{t} C\right)\right)}{W_{2}\left(c_{t}, U^{\infty}\left(S^{t} C\right)\right) \cdot W_{1}\left(c_{t+1}, U^{\infty}\left(S^{t+1} C\right)\right)} .
$$

The stationary (steady state) rate of marginal impatience is defined

\footnotetext{
${ }^{10}$ The rate of interest and rental rate for capital goods are identical in a one-sector model when considered as a competitive equilibrium model. Becker and Boyd [3] discuss this relationship at length.
} 
for the constant sequence $C_{c o n}$ (with each term the scalar $c$ ) by the equation:

$$
1+R\left(C_{c o n}\right)=\frac{1}{W_{2}\left(c, U^{\infty}\left(S C_{c o n}\right)\right)} .
$$

Becker and Boyd [3] recast this by writing $\varrho(c)=R\left(C_{c o n}\right)$ and letting $G^{\infty}(c)=U^{\infty}\left(C_{c o n}\right)=U^{\infty}\left(S C_{c o n}\right)$, so that (8) has the equivalent statement:

$$
1+\varrho(c)=1 / W_{2}\left(c, G^{\infty}(c)\right) .
$$

Here $U^{\infty}\left(\theta_{\text {con }}\right) \geq 0$ implies $G^{\infty}(0) \geq 0$. Examples verify that $U^{\infty}\left(\theta_{\text {con }}\right)>0$ is possible. Moreover, $G^{\infty}$ is increasing and strictly concave in $c .^{11}$

The necessary conditions for the optimal capital sequence $K(k)=\left\{k_{t-1}(k)\right\}$, $0<k_{t-1}<b$ for each $t$, are the Euler equations and feasibility conditions:

$$
\begin{aligned}
1+R\left(S^{t-1} C\right) & =f^{\prime}\left(k_{t}(k)\right), \text { for each } t \geq 1 ; \\
c_{t}(k)+k_{t}(k) & =f\left(k_{t-1}(k)\right), \text { for each } t \geq 1, \\
k_{0}(k) & =k .
\end{aligned}
$$

As previously noted, $0<k_{t-1}(k)<b$ for each $t$ holds together with $c_{t}(k)>0$, so the capital and consumption sequence are regular. The Transversality Condition is:

$$
\lim _{t \rightarrow \infty} U_{t}(C(k)) k_{t}(k)=0 .
$$

The corresponding necessary condition for an optimal stationary program is, $(c, k)$ :

$$
1 / W_{2}\left(c, G^{\infty}(c)\right)=f^{\prime}(k),
$$

and the feasibility condition $h(k)=c$.

The Transversality Condition proven necessary for an optimal path in a recursive utility model depends on the Blackwell aggregator properties in Becker and Boyd's [3] treatment. Their argument does not carry over to the Thompson case due to the spectacular failure of the global Lipschitz conditions embedded in Thompson aggregator functional forms. However, a regular path satisfying the Euler equation and the Transversality Condition is an optimal path does follow for our Thompson case. This sufficiency condition is not special to the Blackwell aggregator preference class. It does, however, turn on the concavity of $U^{\infty}$, which follows from the concavity of the GMM aggregators's functional forms.

The capital stock $k^{*}$ is a stationary optimal capital stock if $K\left(k^{*}\right)=$ $\left\{k^{*}, k^{*}, \ldots\right\}$ is the optimal growth problem's solution for $k_{0}=k^{*}$. This program is both optimal given $k^{*}$, and stationary. The corresponding consumption is denoted $c^{*}=h\left(k^{*}\right)$. We exploit the sufficiency of the Euler and Transversality Conditions for optimality in verifying that the steady state found from the necessary conditions is, in fact, the optimal stationary capital stock.

\footnotetext{
${ }^{11}$ These properties are inherited from the GFP utility as (dropping $\infty$-superscrips to save space), $G(c)=W(c, G(c))=U\left(C_{c o n}\right)=W\left(c . U\left(C_{c o n}\right)\right)$ since $S C_{c o n}=C_{c o n}$. See Appendix 3 for details of $G$ 's properties and the lower solution's (LFP's) corresponding properties, evaluated at stationary consumption sequences.
} 


\subsection{Steady State Theory: A Parametric GMM Example}

The formal proof of the existence of the stationary optimal capital stock is motivated by illustrating it for the special case of the GMM aggregator where $\eta=\rho=\beta=1 / 2$. That is, we temporarily assume

$$
W(x, y)=\sqrt{x}+\frac{1}{2} \sqrt{y} .
$$

Once this case is understood the formal proof of the existence of the stationary optimal program is in reach.

Given this specification of the GMM aggregator, the Monotonicity Theorem in Becker and Boyd ([3], p.168) yields conclusions $(I)$ and $(I I)$ as long as $\partial R / \partial c_{1}<0$, evaluated at $C=\left(c_{1}, S C\right)$, holds. This is the same as verifying the Beals - Koopmans Condition in this application. This means that marginal impatience at time one is diminishing as consumption increases. The recursive and stationary structure of preferences implies this property will also obtain for each future time. Note that $W_{1}\left(c_{t+1}, U^{\infty}\left(S^{t+1} C\right)\right)$ is independent of $c_{t}$. Hence, it suffices to verify this GMM aggregator (12) satisfies the Beals and Koopmans Condition that

$$
\frac{\partial}{\partial x}\left(\frac{W_{1}(x, y)}{W_{2}(x, y)}\right)=-\frac{1}{x} \sqrt{\left(\frac{y}{x}\right)}<0 .
$$

$(I)$ and $(I I)$ immediately follow from the Monotonicity Theorem.

The necessary conditions for a stationary capital stock, $k$, and corresponding consumption, $c$, writing (11) in terms of the capital stock is:

$$
1+\varrho(h(k))=f^{\prime}(k),
$$

where $c=h(k)$. We think of $1+\varrho(h(k))$ as an inverse supply function for capital and $f^{\prime}(k)$ as the inverse demand for capital in a stationary state. The stationary marginal impatience factor $1+\varrho(h(k))$ expresses the planner's willingness to release a marginal unit of current consumption for permanent future consumption in a steady state with capital $k$. By giving up some current consumption the planner supplies more capital (on the margin). The marginal productivity of an additional unit of capital is expressed as the inverse demand for capital in producing more consumption and/or capital goods the following period. Condition (13) says a necessary condition for stationary optimality of $k$ is that the inverse supply and demand for capital are equal. It is reasonable to expect that if the marginal product of capital exceeds the stationary marginal impatience factor then the planner will, in fact, trade some current consumption for additional permanent future consumption (and utility). Similarly if the marginal product of capital is smaller than the stationary marginal impatience factor capital will decumulate (in a steady state).

Given (12) and (9) it follows that $1+\varrho(c)=4 \sqrt{G^{\infty}(c)}$, and for $c=h(k)$,

$$
1+\varrho(f(k)-k)=4 \sqrt{G^{\infty}(h(k))} .
$$


Combining this relation with (13) yields the equivalent steady state condition for our specific choice of the parameters $\beta$ and $\rho$ :

$$
4 \sqrt{G^{\infty}(h(k))}=f^{\prime}(k) .
$$

As noted in Section 5.1 any candidate steady state stock must satisfy $k \leq k^{+}$. Hence, a necessary condition for a steady state stock $k$ is $f^{\prime}(k) \geq 1$. Equation (13) implies $\varrho(f(k)-k) \geq 0$ is also necessary for a steady state stock. In fact, we will show that the Golden-Rule is NOT a stationary optimal program in this example. Hence, $\varrho(f(k)-k)>0$ occurs with a stationary optimal program.

We use a computer algebra system (CAS) and visualization software to illustrate the inverse supply and demand for capital. To do this we must specify two functions: the production function and $G^{\infty}$. Assume production is CobbDouglas:

$$
f(k)=2 \sqrt{k} .
$$

The maximum sustainable stock is $b=4$. The Golden-Rule capital stock is $k^{+}=1$; Golden-Rule consumption is $c^{+}=1$.

Using the Golden-Rule consumption we can visualize the graph of the aggregator (12) with $x=c^{+}=1$. That is, we plot $W\left(c^{+}, y\right)$ as $y$ varies in the functional form (12). Then $W(1, y)=1+\frac{1}{2} \sqrt{y}, y \geq 0$; its graph appears in Figure 1. Notice that there is a unique $y^{+}=W\left(1, y^{+}\right)$and $y^{+}>0$ where the graph of $W(1, y)$ crosses the 45-degree line. This utility level corresponds to the utility level $G^{\infty}\left(c^{+}\right)=y^{+}=W\left(c^{+}, G^{\infty}\left(c^{+}\right)\right)=1.6404$ (using our CAS).

The slope of the tangent line at the fixed point $W\left(1, y^{+}\right)=y^{+}$is clearly smaller than one. That is, $0<W_{2}\left(c^{+}, G_{\infty}\left(c^{+}\right)\right)<1$ at $c^{+}=1$. $W\left(c^{+}, y\right)$ is locally Lipschitz in $y$ at $y=y^{+}$. Indeed, there is a type of local discounting of future utility at the Golden-Rule as $W_{2}\left(1, y^{+}\right) \in(0,1)$. Our CAS calculations yields $W_{2}\left(1, y^{+}\right)=0.19519$. This implies the stationary marginal rate of impatience, measured by

$$
1+\varrho\left(c^{+}\right)=\frac{1}{W_{2}\left(c^{+}, G^{\infty}\left(c^{+}\right)\right)}>1 .
$$

Hence, since $f^{\prime}\left(k^{+}\right)=1$ for the Golden-Rule stock, the steady state condition (15) cannot hold and the Golden-Rule is NOT a discounted Golden-Rule or stationary optimal program.

It turns out that we can solve the functional equation

$$
G(c)=W(c, G(c)) \text { for each } c \geq 0 .
$$

This construction is formalized in Appendix $3 .^{12}$ We note that there is a least (lower) fixed point solution to this equation, denoted by $G_{\infty}(c)$, and it is lower-semicontinuous on $[0, \infty)$, strictly concave, and strictly increasing. By concavity, it is also continuous on $(0, \infty)$. The least fixed point solution to this

\footnotetext{
${ }^{12}$ The restriction $c \in[0,4]$ is not formally required by our solution technique, although the upper bound on consumption from the maximum sustainable stock is required for the general GMM case analyzed in Section 5 .
} 
functional equation is NOT continuous at $c=0$. There is another solution, the greatest (upper) fixed point solution, $G^{\infty}(c)$, which is continuous on $[0, \infty)$. Both solutions agree on $(0, \infty)$, that is: $G_{\infty}(c)=G^{\infty}(c)$ when $c>0$. The functional form for $G_{\infty}$ is:

$$
G_{\infty}(c)=\left\{\begin{array}{c}
\left(\frac{\frac{1}{2}+\sqrt{\left(\frac{1}{2}\right)^{2}+4 \sqrt{c}}}{2}\right)^{2}, \text { if } c>0 ; \\
0 \text { if } c=0 .
\end{array}\right.
$$

The upper solution, $G^{\infty}(c)$, is the function

$$
G^{\infty}(c)=\left(\frac{\frac{1}{2}+\sqrt{\left(\frac{1}{2}\right)^{2}+4 \sqrt{c}}}{2}\right)^{2}, \text { for each } c \geq 0 .
$$

Note the upper solution has the property $G^{\infty}(c)>0$ at $c=0$ whereas the lower solution has downward jump discontinuity at $c=0$. Our computer generated diagrams visualize the upper solution. This should cause no difficulty for the reader in adjusting the figures to visualize the lower solution, which only differs from the upper one at $c=0$.

The graph of $G^{\infty}$ appears in Figure 2. We formally plot the upper solution (dropping the superscript), which we write now as $G(c)$, and label it on the graph's vertical axis by the letter $G$.

Next, set $h(k)=2 \sqrt{k}-k$ and define $S(k)=4 \sqrt{G(h(k))}$. This is the inverse supply of capital. Notice that $h\left(k^{+}\right) \geq h(k)$ for each $k \in[0,4]$ implies $S\left(k^{+}\right) \geq S(k)$ as well! This follows as $G^{\infty}$ and the square root function are strictly increasing. Figure 3 displays the inverse supply function's graph. ${ }^{13}$ The maximum value is achieved at the Golden-Rule stock and that value is denoted $S^{+}=S\left(k^{+}\right)$, with

$$
S(k)=4 \sqrt{\left(\frac{\frac{1}{2}+\sqrt{\left(\frac{1}{2}\right)^{2}+4 \sqrt{h(k)}}}{2}\right)^{2}} .
$$

The value for $S^{+}=S(1)=\sqrt{17}+1=5.1231$ (approximately), as shown in Figure 3.

Figures 4 and 5 clearly show the inverse supply and demand curves intersect at a point $k^{*} \in(0,1)$ with $k^{+}=1$. The equation expressing the excess supply of stationary capital is $S(k)-f^{\prime}(k)=0$. This equation is displayed below for our example.

$$
4 \sqrt{\left(\frac{\frac{1}{2}+\sqrt{\left(\frac{1}{2}\right)^{2}+4 \sqrt{h(k)}}}{2}\right)^{2}}-\frac{1}{\sqrt{k}}=0
$$

\footnotetext{
${ }^{13}$ Plotting the lower solution instead would show the jump discontinuity at the origin, but the graph coincides with the upper soluton for $c$ positive. A shift to the lower solution does not materilly change our analysis showing the existence of the stationary optimal solution since it occurs at a point where the upper and lower solutions agree.
} 
It may be numerically solved; the approximate numerical solution using CAS is: $k^{*}=5.2898 \times 10^{-2}$.

Figure 4 views the inverse supply and inverse demand functions. The latter is $f^{\prime}(k)=1 / \sqrt{k}$. Superimpose these two functions to obtain Figure 4. Note that $S(k)$ has not been defined for $k>4$, the maximum sustainable stock. Figure 5 reimagines Figure 4 with a scale change intended to zoom in on the solution to equation (18). Clearly there is a $k^{*}, 0<k^{*}<k^{+}$with $S\left(k^{*}\right)=f^{\prime}\left(k^{*}\right)$.

The existence and uniqueness of the stationary optimal consumption and capital stock does not change if we shift our focus to the lower solution, $G_{\infty}$, instead of the upper solution favored above. The graphs of the upper and lower solution agree at positive consumption levels where the stationary optimal solution occurs. We note in passing the same is true for the Golden-Rule consumption-capital pair.

\section{Proof of the Turnpike Theorem}

Assume that the aggregator preference relation is given by a GMM Thompson functional form (1) with corresponding utility $U^{\infty}$. There is a unique optimal solution for the optimal growth problem with production function $f$, maximum sustainable stock, $b$, and initial capital $k \in(0, b)$. Moreover, the optimal capital and consumptions sequences are regular. The Beals-Koopmans condition (5) holds:

$$
\begin{aligned}
\frac{\partial}{\partial x}\left(\frac{W_{1}(x, y)}{W_{2}(x, y)}\right) & =\frac{\partial}{\partial x}\left(\frac{\eta \beta_{1} x^{\eta-1}}{\rho \beta_{2} y^{\rho-1}}\right) \\
& =\frac{\left((\eta-1) \eta \beta_{1} x^{\eta-2}\right)}{\rho \beta_{2} y^{\rho-1}}<0 .
\end{aligned}
$$

Therefore the non-crossing and capital monotonicity properties apply to the optimal capital sequence. It remains to prove there is a unique stationary optimal program $k^{*} \in\left(0, k^{+}\right)$where $k^{+}$is the Golden-Rule capital stock. We prove the existence of an optimal stationary program by reasoning in analogy to the graphical constructions in Section 5.3's example.

A necessary condition for $k^{*}$ to be the only stationary optimal capital stock is that it is the unique solution to:

$$
f^{\prime}(k)=1+\varrho(h(k)),
$$

or

$$
f^{\prime}(k)=\frac{1}{W_{2}\left(h(k), G^{\infty}(h(k))\right)} .
$$

If we have a GMM aggregator and its corresponding greatest solution, $G^{\infty}$, evaluated at each stationary consumption, then we can find the righthand side of the previous equation in terms of the GMM parameters. This is exploited to prove that a stationary optimal capital stock exists and it is not the GoldenRule stock. The exact functional form for $G^{\infty}$ is not necessary here to prove 
the proposition below. The result as stated applies to the GFP, $U^{\infty}$. However, the formal proof also applies in the case where the LFP, $U_{\infty}$, replaces the GFP. The reason is that we construct the solution on an interval excluding zero consumption; the LFP and GFP agree on that interval.

Proposition 3 If $W$ is a GMM Thompson aggregator with utility function, $U^{\infty}$, then there is a unique $k^{*} \in\left(0, k^{+}\right)$such that $K\left(k^{*}\right)=\left\{k_{t-1}\left(k^{*}\right)=k^{*}\right\}_{t=1}^{\infty}$ is the stationary optimal capital sequence.

Proof. Let $G^{\infty}(c)=W\left(c, G^{\infty}(c)\right)$ for each $c \geq 0$. For a stationary consumption sequence, the GFP of the Koopmans equation's solution expresses $U^{\infty}\left(C_{c o n}\right)=G^{\infty}(c)=W\left(c, G^{\infty}(c)\right)$ with $c_{t}=c$ for each $t$. The function $G^{\infty}$ is strictly increasing, strictly concave and upper-semicontinuous on $[0, \infty)$. It is also continuous on the open interval $(0, \infty)$ by concavity. Appendix 3 supplies supporting details.

Step 1. The Golden-Rule is not a stationary optimal program.

Observe that $0<W_{2}\left(c^{+}, G^{\infty}\left(c^{+}\right)\right)<1$ since the GMM aggregator's Inada conditions imply there is a unique $y^{+}>0$ such that $W\left(c^{+}, y^{+}\right)=y^{+}$. The slope of the tangent line at $\left(y^{+}, y^{+}\right)$in $\left(y, W\left(c^{+}, y\right)\right)$ - coordinates must be smaller than one by the Inada conditions on $W\left(c^{+}, y\right)$ in $y$ (along with $W\left(c^{+}, 0\right)>0$ and $\left.W_{22}<0\right)$. Hence, it is impossible for the stationary pair $\left(c^{+}, k^{+}\right)$to satisfy the equation $f^{\prime}\left(k^{+}\right)=1 / W_{2}\left(c^{+}, G^{\infty}\left(c^{+}\right)\right)=1$.

Step 2. Define the inverse supply function, $S(k)$, as the stationary marginal rate of impatience (8) in terms of $k$. That is, for $c=h(k)=$ $f(k)-k$ on $[0, b]$ :

$$
S(k)=\frac{1}{W_{2}\left(h(k), G^{\infty}(h(k))\right)}=\frac{1}{\rho \beta_{2}}\left[G^{\infty}(h(k))\right]^{1-\rho} .
$$

This function is strictly concave, increasing on $\left(0, k^{+}\right)$and decreasing on $\left(k^{+}, b\right){ }^{14}$ Its global maximum value, $S^{+}$, occurs when $k=k^{+}$. The Golden-Rule stock has the property $h\left(k^{+}\right) \geq h(k)$ for each $k \in[0, b]$, so $G^{\infty}$ strictly increasing implies $G^{\infty}\left(h\left(k^{+}\right)\right) \geq G^{\infty}(h(k))$ as well. Finally, note that $S^{+}=\left[G^{\infty}\left(h\left(k^{+}\right)\right)\right]^{1-\rho} \geq$ $\left[G^{\infty}(h(k))\right]^{1-\rho}$ also holds as $0<\rho<1$. These inequalities are strict whenever $k^{+} \neq k$.

Step 3. Let $k_{+}=\left(f^{\prime}\right)^{-1}\left(S^{+}\right)$, which is well-defined since $f^{\prime \prime}<0$. Clearly $0<k_{+}$. We claim $k_{+}<k^{+}$. Note that Step 1 yields $S\left(k^{+}\right)>f^{\prime}\left(k^{+}\right)=1$. Apply $\left(f^{\prime}\right)^{-1}$ to both sides of this inequality. Note $f^{\prime \prime}<0$ and the definition of $k_{+}$imply

$$
k_{+}=\left(f^{\prime}\right)^{-1}\left(S\left(k^{+}\right)\right)<\left(f^{\prime}\right)^{-1} f^{\prime}\left(k^{+}\right)=k^{+} .
$$

We can exclude any $k \in\left[0, k_{+}\right]$as a possible solution of the equation $S(k)=$ $f^{\prime}(k)$ since $f^{\prime}(k)>S^{+}$for each $k<k_{+}$. Hence, there is no loss of generality in confining our search for an optimal stationary stock to the interval $\left[k_{+}, k^{+}\right]$. Note: $S(k)$ is continuous on the interval $\left[k_{+}, k^{+}\right]$.

\footnotetext{
${ }^{14} G_{\infty}(c)$ may have a downward jump discontinuity at $k=0$ and $k=b($ as $h(b)=0)$.
} 
Step 4. There exists a unique $k^{*} \in\left(k_{+}, k^{+}\right)$such that $S\left(k^{*}\right)=f^{\prime}\left(k^{*}\right)$. To see this define the excess supply function $\gamma:\left[k_{+}, k^{+}\right] \rightarrow \mathbb{R}$ by

$$
\gamma(k)=S(k)-f^{\prime}(k), k_{+} \leq k \leq k^{+} .
$$

Step 2 yields $S^{+} \geq S(k)$ for each $k \in\left[k_{+}, k^{+}\right]$with strict inequality for $k<k^{+}$. Thus, $\gamma\left(k_{+}\right)<0$ as $S\left(k_{+}\right)-f^{\prime}\left(k_{+}\right)=S\left(k_{+}\right)-S^{+}<0$. Similarly, $\gamma\left(k^{+}\right)>0$ since $S\left(k^{+}\right)-f^{\prime}\left(k^{+}\right)>0$ as $S\left(k^{+}\right)>1=f^{\prime}\left(k^{+}\right)$by Step 1 . This excess supply function is continuous on $\left[k_{+}, k^{+}\right] .{ }^{15}$ Hence, the Intermediate Value Theorem implies there is a capital stock $k^{*} \in\left(k_{+}, k^{+}\right)$such that $\gamma\left(k^{*}\right)=0$.

Step 5. $k^{*}$ is the unique solution to $\gamma(k)=0$ on $\left[k_{+}, k^{+}\right]$. The excess supply function is strictly increasing on $\left[k_{+}, k^{+}\right]$since $S$ is increasing and $f^{\prime \prime}<0$ decreasing on $\left[k_{+}, k^{+}\right]$.

Step 6. $k^{*}$ is the stationary optimal program. This follows since the Transversality Condition (10) takes the special form:

$$
\left(W_{2}\left(c^{*}, G^{\infty}\left(c^{*}\right)\right)\right)^{t} W_{1}\left(c^{*}, G^{\infty}\left(c^{*}\right)\right) k^{*} \rightarrow 0 \text { as } t \rightarrow \infty .
$$

This obtains since $0<W_{2}\left(c^{*}, G^{\infty}\left(c^{*}\right)\right)<1$ with $c^{*}=h\left(k^{*}\right)$. Therefore, the consumption-capital pair $\left(c^{*}, k^{*}\right)$ satisfy the Euler condition (with initial stocks $k_{0}=k^{*}$ ) and the appropriate Transversality Condition, implying $k^{*}$ is the stationary optimal capital stock. Hence, there is a unique stationary optimal capital stock.

The combination of the Beals - Koopmans Condition 3 and Proposition yield the Turnpike Theorem as an application of the Monotonicity and Recursive Turnpike Theorem in Becker and Boyd [3]. The existence and uniqueness of the stationary optimal program implies the planner is discounting future utility in a steady state since $0<W_{2}\left(c^{*}, G^{\infty}\left(c^{*}\right)\right)<1$. For this reason it is reasonable to interpret the steady state as a discounted Golden-Rule program.

\section{Observational Equivalence of Optimal Growth with Extremal Objectives}

The Turnpike Theorem implies that the optimal capital and consumption sequences are elements of $\ell_{\infty}^{++}$. Both are clearly bounded sequences since they are convergent sequences. It is also clear that the $\inf _{t} c_{t}(k)>0$, so $C(k) \in \ell_{\infty}^{++}$. Uniqueness theory for the Koopmans equation yields the LFP and GFP agree on the norm-interior of the commodity space's positive cone. Hence, for EACH $C \in \ell_{\infty}^{++}$, we observe that $U_{\infty}(C)=U^{\infty}(C)$. Counterexamples show that $U_{\infty}(\theta)<U^{\infty}(\theta)$ can obtain. Hence, we can only say that $U_{\infty}(C) \leq U^{\infty}(C)$ obtains on the boundary, $\partial \ell_{\infty}^{+}$. The main observation is that the optimal solution to the problem $\mathcal{P}^{\infty}(k)$ yields $U_{\infty}(C(k))=U^{\infty}(C(k))=J^{\infty}(k)$. Since the solution to $\mathcal{P}^{\infty}(k)$ is unique (by $U^{\infty}$ strictly concave), it is reasonable

\footnotetext{
${ }^{15}$ For the case of $G_{\infty}$, jump discontinuities, if any, occur at either $k=0$ or $k=b$ and are excluded from the excess supply function's domain.
} 
to conjecture $J_{\infty}(k)=U_{\infty}(C(k))=U^{\infty}(C(k))=J^{\infty}(k)$ and $C(k)$ also solves $\mathcal{P}_{\infty}(k)$. In fact, this observation carries over to any $U \in \operatorname{fix}\left(T_{W}\right)$ since $U_{\infty}(C) \leq U(C) \leq U^{\infty}(C)$ for each $C \in \ell_{\infty}^{+}$. The theoretical results underpinning these observations and conjectures are derived from the general existence and uniqueness results for the Koopmans operator with Thompson aggregators covered in Becker and Rincón-Zapatero ([4], [5]), and Marinacci and Montrucchio ([19], [20]). Note that the ONLY difference between the optimal growth problems $\mathcal{P}_{U}(k)$ is the specification of the objective functions, which differ only on the boundary of the positive cone of $\ell_{\infty}^{+}$. The feasible consumption and capital sequences remain the same as the initial capital stock remains the same when specifying $\mathcal{P}_{U}(k)$.

The main result showing the optimal growth solution does NOT depend on the choice of $U \in \operatorname{fix}\left(T_{W}\right)$ is:

Theorem 4 (Observational Equivalence with Extremal Objectives). Let $C(k)=$ $\arg \max _{C}\left\{U^{\infty}(C): C \in F(k)\right\}$ be the unique solution to $\mathcal{P}^{\infty}(k)$. Then for each $U \in \operatorname{fix}\left(T_{W}\right), C(k)=\arg \max _{C}\{U(C): C \in F(k)\}$ is the unique solution to $\mathcal{P}_{U}(k)$. In particular, $C(k)=\arg \max _{C}\left\{U_{\infty}(C): C \in F(k)\right\}$ is the unique solution to $\mathcal{P}_{\infty}(k)$.

This Theorem's implication is that the Turnpike Theorem holds for the solution found for each possible choice of $U \in \operatorname{fix}\left(T_{W}\right)$. Indeed, the optimal solution is the same for each such utility function. The boundary elements, consumption sequences in $\partial \ell_{\infty}^{+}$, never appear in the optimal consumption sequence! Hence, each potential objective $U \in$ fix $\left(T_{W}\right)$ has the same optimal value and same consumption sequence which achieves that value. The calculation of an optimum or characterizing the optimum's properties may be established using whichever objective in fix $\left(T_{W}\right)$ is most easily adapted to the analytic arguments necessary to prove a property.

Proof. The strict concavity of $U^{\infty}$ implies the optimal solution to $\mathcal{P}^{\infty}(k)$, denoted $C(k)$, is unique. Hence, we immediately verify by standard uniqueness theory for Thompson aggregators (see above references):

Step 1. $U_{\infty}(C(k))=U^{\infty}(C(k))=J^{\infty}(k)$.

Step 2. $J_{\infty}(k) \geq U_{\infty}(C(k))$ since $C(k)$ is a feasible consumption sequence for $\mathcal{P}_{\infty}(k)$.

Step 3. $J_{\infty}(k)=J^{\infty}(k)$. Step 1 yields $U_{\infty}\left(C(k)=U^{\infty}(C(k))=J^{\infty}(k)\right.$. Since $U_{\infty}(C) \leq U^{\infty}(C)$ for each $C \in[0, b]^{\infty}$, it follows that

$$
J_{\infty}(k)=\sup \left\{U_{\infty}(C): C \in F(k)\right\} \leq \sup \left\{U^{\infty}(C): C \in F(k)\right\}=J^{\infty}(k) .
$$

Steps 1 and 2 imply

$$
U_{\infty}(C) \leq J_{\infty}(k) \leq J^{\infty}(k)=U^{\infty}(C(k))=U_{\infty}(C(k)) \leq J_{\infty}(k),
$$

and thus equality holds and we deduce $J_{\infty}(k)=J^{\infty}(k)$.

Step 4. $C(k)=\arg \max \left\{U_{\infty}(C): C \in F(k)\right\}$. We know that $C(k) \in$ $\arg \max \left\{U_{\infty}(C): C \in F(k)\right\}$ by the previous step. We only know that $U_{\infty}(C)$ 
is a concave function on $[0, b]^{\infty}$. So, we may have another optimal solution for $\sup \left\{U_{\infty}(C): C \in F(k)\right\}$. If so, that solution, denoted by $C^{*}(k)$, must achieve the same value: that is, $U_{\infty}\left(C(k)=U_{\infty}\left(C^{*}(k)\right)=J_{\infty}(k)\right.$. In fact, that distinct solution must be an element of $\ell_{\infty}^{++}$as well. ${ }^{16}$ So, it follows that $U^{\infty}(C(k))=U^{\infty}\left(C^{*}(k)\right)$ must also hold since $C^{*}(k)$ is feasible in $\mathcal{P}^{\infty}(k)$. But $U^{\infty}$ is strictly concave on $[0, b]^{\infty}$. A strict convex combination of the sequence $C(k)$ and $C^{*}(k)$ must be feasible and yield a higher utility payoff for $\mathcal{P}^{\infty}(k)$ than either $U^{\infty}(C(k))$ or $U^{\infty}\left(C^{*}(k)\right)$, a contradiction to the uniqueness of the optimal solution to $\mathcal{P}^{\infty}(k)$.

Step 5. Now consider any $U \in \operatorname{fix}\left(T_{W}\right)$. By $U_{\infty} \leq U \leq U^{\infty}$ it must be the case that $U_{\infty}(C(k))=U(C(k))=U^{\infty}(C(k))$. It now follows that $C(k)$ solves $\mathcal{P}_{U}(k)$ as well. In fact, this is the only possible solution to $\mathcal{P}_{U}(k)$. Suppose there are two distinct solutions to $\mathcal{P}_{U}(k)$, then each must offer the same utility payoff. Observe $J_{U}(k)=J_{\infty}(k)=J^{\infty}(k)$ (by following the previous steps again). Hence, both solutions can be considered in a convex combination of feasible consumption programs given $k$. Then this convex combination is feasible for $\mathcal{P}^{\infty}(k)$ and must yield a strictly larger value than $U^{\infty}(C(k))=J^{\infty}(k)$ as well since $U^{\infty}$ is a strictly concave function on $[0, b]^{\infty}$, contradicting the uniqueness of the solution to $\mathcal{P}^{\infty}(k)$.

\section{Conclusion}

The Turnpike Theorem shows that it is possible to carry out a qualitative analysis of optimal capital sequences for the GMM Thompson aggregators. Specifically, the standard monotonicity and non-crossing properties obtain. The optimal capital sequence converges to the long-run stationary or steady state capital stock as well. That steady state, or discounted Golden-Rule, is uniquely determined given properties on technology and the GMM aggregator alone.

Our Observational Equivalence result shows the importance of the aggregator's preference structure for analyzing the properties of optimal growth paths. Aggregator preferences alone determine the actual qualitative features of optimal consumption and capital sequences since we do not have explicit functional forms for the underlying extremal utility functions. This links back to the aggregator based sufficient conditions first offered by Beals and Koopmans [6]. Their strategy for proving, for example, monotonicity of the optimal capital sequence rests on assumed properties of a given aggregator preference, which turn out to be verifiable in our GMM Thompson specifications. Given there are extremal solutions to the Koopmans equation, it is of some interest to conclude that the choice of a particular solution to that equation is only important in so far as some qualitative properties are more readily proven for the Koopmans operator's Greatest Fixed Point rather than its Least Fixed Point. Two examples

\footnotetext{
${ }^{16}$ Inada conditions on the utility function, as before, with a positive initial capital stock, insures that the optimal path is regular. The Turnpike Theorem applies to this optimal path with the objective $U_{\infty}$ instead of $U^{\infty}$. The stationary optimal program is the same for both objectives as well.
} 
illustrate this point: the strictly concavity of the GFP and the upper semicontinuity of that utility function in the product topology for the commodity space are easier to demonstrate directly than for the corresponding LFP.

The GMM family does not contain all the known Thompson aggregator examples. For example, it would be interesting to reexamine our results for the Koopmans-Diamond-Williamson (KDW) [16] aggregators that are outside the Blackwell class. It is known a global Lipschitz condition in the aggregator's second argument holds for all KDW aggregators, but that global Lipschitz constant is larger than (or equal to) one in the Thompson case. The Blackwell requirement is that constant be positive and strictly less than one. A parametric representation of the KDW aggregator takes it outside the Blackwell family. For example, the Blackwell KDW aggregators (with Lipschitz constant strictly smaller than one) exhibit at most one steady state. See Becker and Boyd ([3], p. 161) for details. However, it is not yet known if a steady state exists for the corresponding Thompson aggregator. We conjecture that it will be possible to replicate our results in the Thompson KDW case. Indeed, preliminary CAS computations yield examples of particular KDW aggregators with local Lipschitz constants less than one at potential steady states in future utility and aggregator value coordinates for a given first period's consumption (analogous to our Figure 1).

An extension of our results to two-sector models after the treatment by Benhabib, Majumdar and Nishimura [7] seems in reach as their results rely on Beals-Koopmans type conditions for their monotonicity results. The ana$\log$ of that condition is linked to the transformation function representing the two-sector technology in their setup. There is also the potential for multiple steady state solutions that might create multiple "basins of attraction," with initial conditions determining which steady state is the long-run attractor for the optimal capital sequences.

An important open question revolves around extending the turnpike theory to one-sector models capable of sustained or endogenous growth. For example, is there a stationary optimal capital stock when the production function is an $A k$ function $(A>1)$ ? There is no Golden-Rule when technology is $A k$, so different techniques may be necessary to study optimal capital accumulation with utility derived from a Thompson aggregator.

\section{Appendices:}

\subsection{Appendix 1: Recovering Utility Functions from a GMM Aggregator}

Thompson aggregators can be found by iteration of the Koopmans operator for suitable initial seeds. This successive approximation technique does not, in general, yield a unique fixed point for Thompson recursive utility on the entire commodity space domain, $\ell_{\infty}^{+}$. While general recovery theory of extremal utility functions defined on $\ell_{\infty}^{+}$may be found by application of monotone operator 
methods, we focus on recovery of extremal utility functions given an aggregator by restricting consumption sequences to the set $[0, b]^{\infty} \subset \ell_{\infty}^{+}$, where $b=f(b)>$ 0 is the economy's maximum sustainable capital stock. Let $y^{b}=W\left(b, y^{b}\right)$ define a utility level when consumption at time one is $b$ and the continuation utility is $y^{b}$. The existence of a continuation utility value is a consequence of the postulates defining a concave Thompson aggregator in general, and our CES aggregator functional form, in particular. Let $T_{W}$ denote the Koopmans operator mapping the order interval of bounded functions defined on $[0, b]^{\infty}$ to itself. Let $B^{+} \equiv B^{+}\left([0, b]^{\infty}, \mathbb{R}_{+}\right)$be the bounded nonnegative functions defined on $[0, b]$.with its usual pointwise partial order. The order interval contained in this set is $\left\langle\theta, y^{b}\right\rangle$ where $\theta$ is the zero function and $y^{b}$ (abusing notation) is the constant function $y^{b}(C)=y^{b}=W\left(b, y^{b}\right)$ for each sequence $C \in[]^{\infty}$. That is, $0 \leq U(C) \leq y^{b}$ for each $C \in[0, b]^{\infty}$. The possible utility functions in the order interval $\left\langle\theta, y^{b}\right\rangle$ have the common upper bound, $y^{B}$.

For $U \in\left\langle\theta, y^{b}\right\rangle, T_{W} U(C)=W\left(c_{1}, U(S C)\right)$, where $S$ is the shift operator on $[0, b]^{\infty}$. Note that $T_{W} U \in\left\langle\theta, y^{b}\right\rangle$. Define the iterates (for each natural number $N)$ :

$$
T_{W}^{N} U(C)=W\left(c_{1}, W\left(c_{2}, \ldots, W\left(c_{N}, U\left(S^{N}(C)\right)\right) \ldots\right)\right),
$$

where $S^{N}(C)=\left\{c_{N+1}, c_{N+2}, \ldots\right\}$. Evidently $T_{W}^{N} U \in\left\langle\theta, y^{b}\right\rangle$. Consider two choices of $U$ : the bottom element of $\left\langle\theta, y^{b}\right\rangle, \theta$, and the top element, $y^{b}$. Initiating the iteration at $U=\theta$ yields $T_{W} \theta(C)=W\left(c_{1}, \theta\right), T_{W}^{2} \theta(C)=W\left(c_{1}, W\left(c_{2}, \theta\right)\right)$ and so on. Likewise, $T_{W} y^{b}(C)=W\left(c_{1}, y^{b}\right), T_{W}^{2}\left(c_{1}, W\left(c_{2}, y^{b}\right)\right)$ and so on. The following observations follow from the general Thompson recovery theory as exposited by Becker and Rincón-Zapatero [4]: there exist functions $U_{\infty}$ and $U^{\infty}$ in $\left\langle\theta, y^{b}\right\rangle$ such that for each $C \in[0, b]^{\infty}$ :

1. $T_{W}^{N} \theta(C) \nearrow U_{\infty}(C)$ and $T_{W}^{N} y^{b}(C) \searrow U^{\infty}(C)$;

2. $T_{W} U_{\infty}(C)=U_{\infty}(C)$ and $T_{W} U^{\infty}(C)=U^{\theta}(C)$;

3. $U_{\infty}(C) \leq U^{\infty}(C)$;

4. $U_{\infty}\left(U^{\infty}\right)$ is the Least (Greatest) Fixed Point of $T_{W}$.

Observe that both $\theta$ and $y^{b}$ are continuous real-valued functions on $[0, b]$, so it follows that $T_{W} \theta$ is as well in $B^{+}$, and so on for each iterate. Note that each $T_{W}^{N} \theta$ is a norm continuous function in $B_{+}$. In fact, more importantly for proving Theorem 1, it is also continuous in the product topology which is the topology of coordinatewise convergence in $B_{+}$. Hence, we conclude that $U_{\infty}$ is a lower-semicontinuous function in $B^{+}$with its relative product topology as the pointwise supremum of the sequence of product continuous functions, $T_{W}^{N} \theta$. Similar reasoning yields $U^{\infty}$ is upper semi-continuous in the relative product topology of $B_{+}$as the pointwise infimum of the sequence of product continuous functions $T_{W}^{N} y^{b}$.

Properties (1)-(4) are consequences of proving the Koopmans operator is $\sigma-$ order continuous so that application of the Tarski-Kantorovich Fixed Point 
Theorem (see Granas and Dugundji [11] and Tarski [21]) is viable. The applicability of the Tarski-Kantorovich Theorem is shown in Becker and RincónZapatero [4]. Balbus, et al [2] also present a version of the Tarski-Kantorovich Theorem that recasts the order continuity property of a nonlinear operator as one preserving monotonic sups and infs of sequence of functions. This latter form of order continuity is the form we verify in the existence (recovery) results quoted from Becker and Rincón-Zapatero [4].

\subsection{Appendix 2: Proof that the GFP of the Koopmans Equation is Strictly Concave}

The GFP $U^{\infty}$ may be shown to be a concave function on $[0, b]^{\infty}$ : just note that each iterate $T_{W}^{N} y^{b}$ is a concave function on that domain by induction. That is, for $N=1, T_{W} y^{b}(C)=W\left(c_{1}, y^{b}(S C)\right)=W\left(c_{1}, y^{b}\right)$ is jointly concave in its arguments. A routine induction step yields concavity of $T_{W}^{N} y^{b}$ on $[0, b]^{\infty}$. Just follow the basic arguments verifying the LFP is concave in Becker and RincónZapatero [4]. The purpose of this appendix is to prove that $U^{\infty}$ is strictly concave on $[0, b]^{\infty}$.

Let $W$ be an aggregator, $W: \mathbb{R}_{+} \times[0, b] \longrightarrow[0, b]$ satisfying properties $1-$ 3 below:

1. $W$ is increasing separately in variables $x$ and $y$;

2. $W$ is concave;

3. For all $\lambda \in(0,1)$, for all $y, y^{\prime} \in[0, b], x, x^{\prime} \in \mathbb{R}_{+}, x \neq x^{\prime}$ implies

$$
W\left(\lambda x+(1-\lambda) x^{\prime}, \lambda y+(1-\lambda) y^{\prime}\right)>\lambda W(x, y)+(1-\lambda) W\left(x^{\prime}, y^{\prime}\right) .
$$

The third property is weaker than strict concavity in $(x, y)$. The aggregator $W(x, y)=\beta_{1} x^{\eta}+\beta_{2} y^{\rho}$ with $0<\beta_{1}, \beta_{2}$ and $0<\eta, \rho<1$ is strictly concave, and hence satisfies 3. Likewise for the KDW aggregator $W(x, y)=$ $(\delta / d) \log \left(1+a c^{b}+d y\right)$ with $a, b, d, \delta>0$ and $b<1$. However, the TAS aggregator $W(x, y)=u(x)+\beta y$, with $u$ strictly concave is not strictly concave in $(x, y)$, but still satisfies property 3 .

We introduce some useful notation next. Given $t \geq 1$, let $C_{t}=\left(c_{1}, \ldots, c_{t}\right)$, where $c_{t} \geq 0 .{ }^{17}$ Note that $C_{1}=c_{1}$. Define the function $W^{t}: \mathbb{R}_{+}^{t} \times[0, b] \longrightarrow[0, b]$ recursively as follows

$$
\begin{aligned}
W^{1}\left(c_{1}, y\right) & =W\left(c_{1}, y\right) \\
W^{t}\left(c_{1}, \ldots, c_{t}, y\right) & =W^{t-1}\left(c_{1}, \ldots, c_{t-1}, W\left(c_{t}, y\right)\right), \quad t>1 .
\end{aligned}
$$

We simplify our notation further by setting $C_{t}^{\lambda}=\lambda C_{t}+(1-\lambda) C_{t}^{\prime}, y^{\lambda}=$ $\lambda y+(1-\lambda) y^{\prime}$ and $c_{t}^{\lambda}=\lambda c_{t}+(1-\lambda) c_{t}^{\prime}$.

\footnotetext{
${ }^{17}$ The use of an upper case $C_{t}$ here is not to be confused with our use of upper case $C$ to denote the sequence $\left\{c_{t}\right\}=\left\{c_{t}\right\}$ used in the main body of the text.
} 
Claim 1. For any $t \geq 1, W^{t}$ is increasing in $y$.

Apply mathematical induction. It is true for $t=1$, by property 1 . Suppose that it is true for $t$ and let us prove it for $t+1$. Let $C_{t+1} \in \mathbb{R}_{+}^{t+1}$ and let $y_{1}, y_{2} \in[0, b]$, with $y_{1}<y_{2}$. Then $W\left(c_{t+1}, y_{1}\right)<W\left(c_{t+1}, y_{2}\right)$ by property 1 . By the induction hypotheses, $W^{t}$ is increasing in $y$, hence $W^{t}\left(C_{t}, W\left(c_{t+1}, y_{1}\right)\right)<$ $W^{t}\left(C_{t}, W\left(c_{t+1}, y_{2}\right)\right)$, but this is the same as $W^{t+1}\left(C_{t+1}, y_{1}\right)<W^{t+1}\left(C_{t+1}, y_{2}\right)$, by definition of $W^{t+1}$.

Claim 2. For any $t \geq 1, W^{t}$ is concave in $\left(C_{t}, y\right)$.

Apply mathematical induction. Claim 2 is true for $t=1$ by property 2 . Suppose that it is true for $t$; we prove it for $t+1$.

Let $C_{t+1}, C_{t+1}^{\prime} \in \mathbb{R}_{+}^{t+1}, \lambda \in[0,1]$. The concavity of $W$ (property 2) yields $W\left(c_{t+1}^{\lambda}, y^{\lambda}\right) \geq \lambda W\left(c_{t+1}, y\right)+(1-\lambda) W\left(c_{t+1}^{\prime}, y^{\prime}\right)$. Since $W^{t}$ is increasing in variable $y$ by Claim 1, we have

$$
\begin{aligned}
W^{t}\left(C_{t}^{\lambda}, W\left(c_{t+1}^{\lambda}, y^{\lambda}\right)\right) & \geq W^{t}\left(C_{t}^{\lambda}, \lambda W\left(c_{t+1}, y\right)+(1-\lambda) W\left(c_{t+1}^{\prime}, y^{\prime}\right)\right) \\
& =W\left(\lambda\left(C_{t}, W\left(c_{t+1}, y\right)\right)+(1-\lambda)\left(C_{t}^{\prime}, W\left(c_{t+1}^{\prime}, y^{\prime}\right)\right)\right) \\
& \geq \lambda W^{t}\left(C_{t}, y\right)+(1-\lambda) W^{t}\left(C_{t}^{\prime}, y^{\prime}\right),
\end{aligned}
$$

where the second inequality follows from the induction hypothesis. But by definition of $W^{t+1}$, this is equivalent to

$$
W^{t+1}\left(C_{t+1}^{\lambda}, y^{\lambda}\right) \geq \lambda W^{t+1}\left(C_{t+1}, y\right)+(1-\lambda) W^{t+1}\left(C_{t+1}^{\prime}, y^{\prime}\right) .
$$

Claim 3. Let $C_{t}, C_{t}^{\prime} \in \mathbb{R}_{+}^{t}$ such that $c_{t} \neq c_{t^{\prime}}$. Let $\lambda \in(0,1)$ and let $y, y^{\prime} \in$ $[0, b]$. Then

$$
W^{t}\left(\lambda C_{t}+(1-\lambda) C_{t}^{\prime}, \lambda y+(1-\lambda) y^{\prime}\right)>\lambda W^{t}\left(C_{t}, y\right)+(1-\lambda) W^{t}\left(C_{t}^{\prime}, y^{\prime}\right) .
$$

Apply mathematical induction. Let $t=1$. The inequality (21) is

$$
W^{1}\left(C_{1}^{\lambda}, y^{\lambda}\right)>\lambda W^{1}\left(C_{1}, y\right)+(1-\lambda) W^{1}\left(C_{1}^{\prime}, y^{\prime}\right),
$$

which is true by property 3. Suppose that it is true for $t$ and let us prove it for $t+1$. Let $C_{t+1}, C_{t+1}^{\prime} \in \mathbb{R}_{+}^{t+1}$ such that $c_{t+1} \neq c_{t^{\prime}+1}, \lambda \in(0,1)$ and $y, y^{\prime} \in[0, b]$. By property 3 we have

$$
W\left(c_{t+1}^{\lambda}, y^{\lambda}\right)>\lambda W\left(c_{t+1}, y\right)+(1-\lambda) W\left(c_{t+1}^{\prime}, y^{\prime}\right) .
$$

Now, Claim 1 implies $W^{t}$ is increasing in its $y$ argument. Therefore,

$$
\begin{aligned}
W^{t}\left(C_{t}^{\lambda}, W\left(c_{t+1}^{\lambda}, y^{\lambda}\right)\right. & >W^{t}\left(C_{t}^{\lambda}, \lambda W\left(c_{t+1}, y\right)+(1-\lambda) W^{t}\left(C_{t}^{\prime}, y^{\prime}\right)\right) \\
& \geq \lambda W^{t}\left(C_{t}, W\left(c_{t+1}, y\right)\right)+(1-\lambda) W\left(C_{t}^{\prime}, W\left(c_{t+1}^{\prime}, y^{\prime}\right)\right),
\end{aligned}
$$

where the second inequality follows from Claim 2. Thus, by the definition of $W^{t+1}$

$W^{t+1}\left(\lambda C_{t+1}+(1-\lambda) C_{t+1}^{\prime}, \lambda y+(1-\lambda) y^{\prime}\right)>\lambda W^{t+1}\left(C_{t+1}, y\right)+(1-\lambda) W^{t+1}\left(C_{t+1}^{\prime}, y^{\prime}\right)$ and we are done. Claims $1-3$ prepare the proof of the following lemma. 
Lemma $5 U^{\infty}$ is strictly concave.

Proof. Let $C, C \in[0, b]^{\infty}$, with $C \neq C^{\prime}$. Then there is $t \geq 1$ such that $c_{t} \neq c_{t}^{\prime}$. Let $\lambda \in(0,1)$ and form the convex combination $C^{\lambda}=\lambda C+(1-\lambda) C^{\prime}$. Then

$$
U^{\infty}\left(C^{\lambda}\right)=W\left(c_{1}^{\lambda}, U^{\infty}\left(S C^{\lambda}\right)\right)
$$

and after $t$ iterations we obtain (recall the definition of $W^{t}$ )

$U^{\infty}\left(C^{\lambda}\right)=W^{t}\left(C_{t}^{\lambda}, U^{\infty}\left(S^{t+1} C^{\lambda}\right)\right) \geq W^{t}\left(C_{t}^{\lambda}, \lambda U^{\infty}\left(S^{t+1} C\right)+(1-\lambda) U^{\infty}\left(S^{t+1} C^{\prime}\right)\right)$, since $U^{\infty}$ is concave, and $W^{t}$ is increasing in $y$ (by Claim 1).

Apply Claim 3 with $y=U^{\infty}\left(S^{t+1} C\right)$ and $y^{\prime}=U^{\infty}\left(S^{t+1} C^{\prime}\right)$, since $c_{t} \neq c_{t}^{\prime}$, we obtain

$$
U^{\infty}\left(C^{\lambda}\right)>\lambda W^{t}\left(C_{t}, U^{\infty}\left(S^{t+1} C\right)\right)+(1-\lambda) W^{t}\left(C_{t}^{\prime}, U^{\infty}\left(S^{t+1} C^{\prime}\right)\right)
$$

but $W^{t}\left(C_{t}, U^{\infty}\left(S^{t+1} C\right)\right)=U^{\infty}(C)$ and $W^{t}\left(C_{t}^{\prime}, U^{\infty}\left(S^{t+1} C^{\prime}\right)\right)=U^{\infty}\left(C^{\prime}\right)$, hence

$$
U^{\infty}\left(C^{\lambda}\right)>\lambda U^{\infty}(C)+(1-\lambda) U^{\infty}\left(C^{\prime}\right)
$$

\subsection{Appendix 3: Solving $G(c)=W(c, G(c))$ and an Exam- ple}

The functional equation $G(c)=W(c, G(c))$ governing the utility for constant consumption sequences may be studied with methods analogous to those in Appendix 1. There are extremal solutions: $G^{\infty}(c) \geq G_{\infty}(c) \geq 0$ which are increasing and strictly concave on $[0, b]$. The upper solution, $G^{\infty}$, is upper semicontinuous and the lower solution, $G_{\infty}(c)$, is lower semicontinuous on $[0, b]$. It turns out that $G^{\infty}(c)=G_{\infty}(c)$ whenever $c>0$.

Our example of the solutions to this functional equation is developed below. The example yields $G^{\infty}(0)>G_{\infty}(0)=0$. Consider the aggregator:

$$
W(x, y)=\beta_{1} x^{\eta}+\beta_{2} \sqrt{y},
$$

with $0<\beta_{1}, \beta_{2}, \eta<1$. It is a $M M$ Thompson aggregator with $\rho=1 / 2$. Any function satisfying $G(c)=W(c, G(c))$ for this aggregator satisfies the equation:

$$
\beta_{1} x^{\eta}+\beta_{2} \sqrt{y}=y,
$$

or the equivalent equation

$$
y-\beta_{1} x^{\eta}+\beta_{2} \sqrt{y}=0 .
$$

Make the change of variables $z^{2}=y$ and rewrite the last line as the quadratic equation in $z$ :

$$
z^{2}-\beta_{2} z-\beta_{1} x^{\eta}=0
$$


We show this change of variables introduces a spurious solution to this quadratic equation which allows us to find the distinct upper and lower solutions on the domain $[0, b]$. In fact, our solutions hold on the larger domain $[0, \infty)$.

The quadratic equation has two solutions:

$$
z_{ \pm}=\frac{\beta_{2} \pm \sqrt{\beta_{2}^{2}+4 \beta_{1} x^{\eta}}}{2}
$$

Rewriting the previous line in terms of the variable $y$ we obtain two possibilities:

$$
y_{ \pm}=\left(\frac{\beta_{2} \pm \sqrt{\beta_{2}^{2}+4 \beta_{1} x^{\eta}}}{2}\right)^{2} \text {. }
$$

Substituting $y_{+}$into (23) by identifying $G^{\infty}(c)=y_{+}(c)$ for $c \geq 0$, we verify this function satisfies (23).

The same is not true for $y_{-}$when it is substituted into (23). Notice that $\sqrt{\beta_{2}^{2}+4 \beta_{1} x^{\eta}} \geq \beta_{2}$. We see that

$$
\sqrt{y_{-}}=\left|\frac{\beta_{2}-\sqrt{\beta_{2}^{2}+4 \beta_{1} x^{\eta}}}{2}\right|=\frac{\sqrt{\beta_{2}^{2}+4 \beta_{1} x^{\eta}}-\beta_{2}}{2} .
$$

Substitute the last expression into the left hand side of (22) and obtain:

$$
\begin{aligned}
\beta_{1} x^{\eta}+\beta_{2} \sqrt{y_{-}} & =\beta_{1} x^{\eta}+\beta_{2}\left(\frac{\sqrt{\beta_{2}^{2}+4 \beta_{1} x^{\eta}}-\beta_{2}}{2}\right) \\
& =\beta_{1} x^{\eta}-\frac{\beta_{2}^{2}}{2}+\beta_{2}\left(\frac{\sqrt{\beta_{2}^{2}+4 \beta_{1} x^{\eta}}}{2}\right)
\end{aligned}
$$

while the right hand side becomes

$$
\begin{aligned}
y_{-} & =\left(\frac{\beta_{2}-\sqrt{\beta_{2}^{2}+4 \beta_{1} x^{\eta}}}{2}\right)^{2} \\
& =\frac{\beta_{2}^{2}}{4}-\beta_{2}\left(\frac{\sqrt{\beta_{2}^{2}+4 \beta_{1} x^{\eta}}}{2}\right)+\frac{\beta_{2}^{2}+4 \beta_{1} x^{\eta}}{4} \\
& =\frac{\beta_{2}^{2}}{2}-\beta_{2}\left(\frac{\sqrt{\beta_{2}^{2}+4 \beta_{1} x^{\eta}}}{2}\right)+\beta_{1} x^{\eta}
\end{aligned}
$$

A comparison of (24) and (25) shows they differ for each $x>0$ and agree at $x=0$. Consequently, $y_{-}$is NOT a solution to (22). Note however there are two solutions to $(22)$ when $x=0$ - namely, $y_{-}(0)=0$ and $y_{+}(0)=\beta_{2}^{2}>0$. This exceptional situation allows us to construct two globally different solutions which coincide for each $c>0$ and differ only when $c=0$. Formally, we observe 
that $G^{\infty}$ and $G_{\infty}$ may be defined by the formulas given below for each $c \geq 0$. First, let

$$
G^{\infty}(c)=y_{+}(c)=\left(\frac{\beta_{2}+\sqrt{\beta_{2}^{2}+4 \beta_{1} x^{\eta}}}{2}\right)^{2}
$$

and

$$
G_{\infty}(c)=\left\{\begin{array}{c}
0, \text { if } c=0 \\
y_{+}(c), \text { if } c>0 .
\end{array}\right.
$$

Clearly both satisfy our functional equation, $G^{\infty}(c) \geq G_{\infty}(c) \geq 0$ with $G^{\infty}(c)=$ $G_{\infty}(c)$ for each $c>0$ and $\beta_{2}^{2}=G^{\infty}(0)>G_{\infty}(0)=0$. Both solutions are strictly concave and increasing functions on $[0, \infty) . G^{\infty}$ turns out to be continuous on that domain while $G_{\infty}$ is lower semicontinuous at $c=0$ and continuous for each $c>0$. 


\section{Figures}

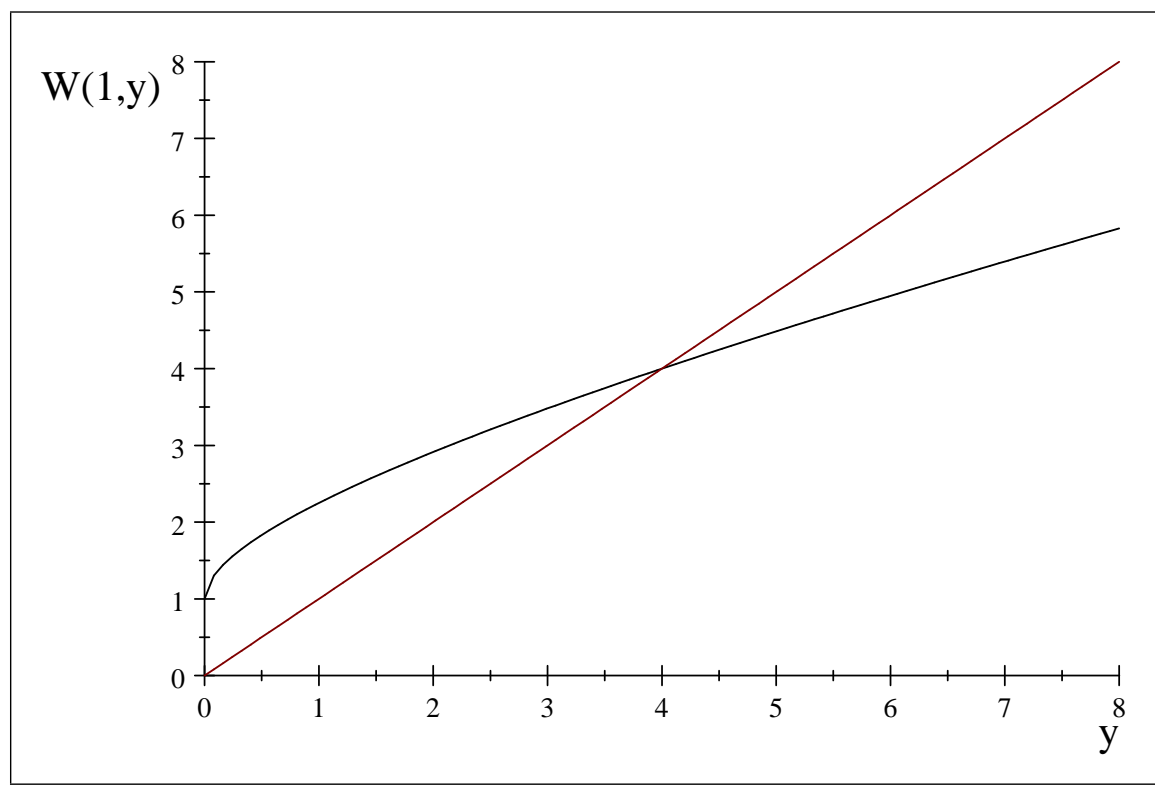

Figure 1: The graph of

$$
W(1, y)=1+\frac{1}{2} \sqrt{y} .
$$

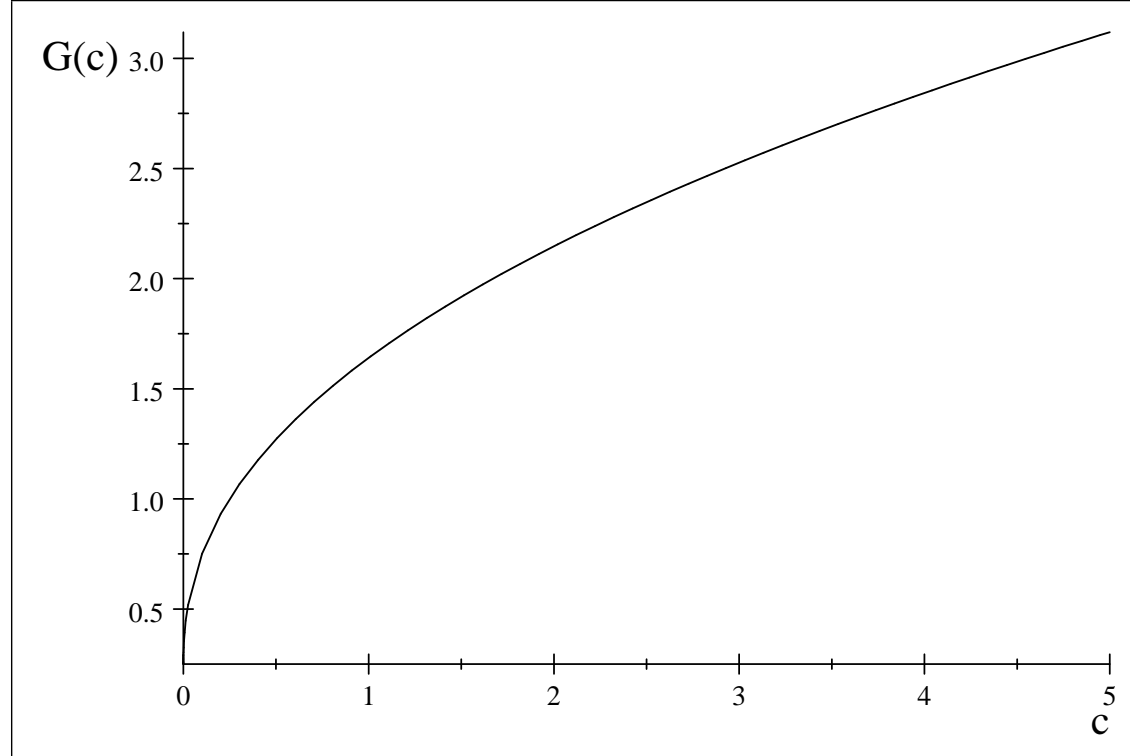

Figure 2: The Upper Solution $G^{\infty}(c) ; G^{\infty}(0)>0$. 


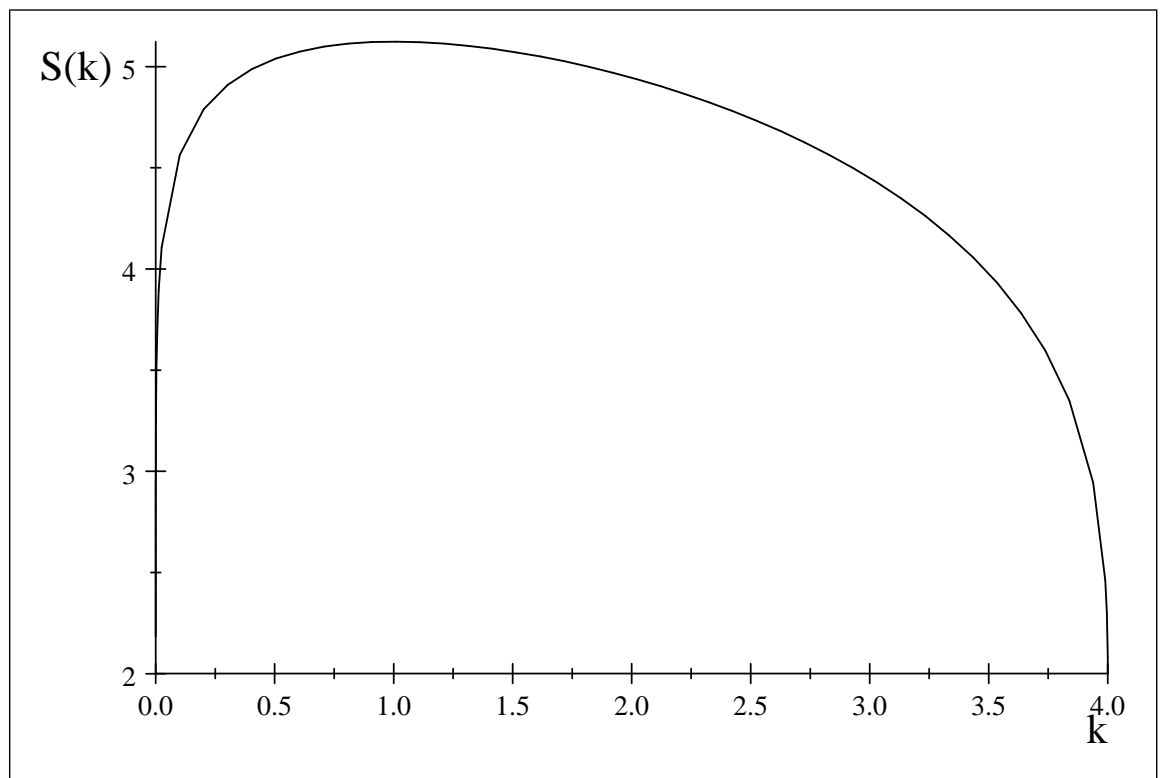

Figure 3: The Inverse Supply Function with Maximum Value at the Golden-Rule, $k^{+}=1$.

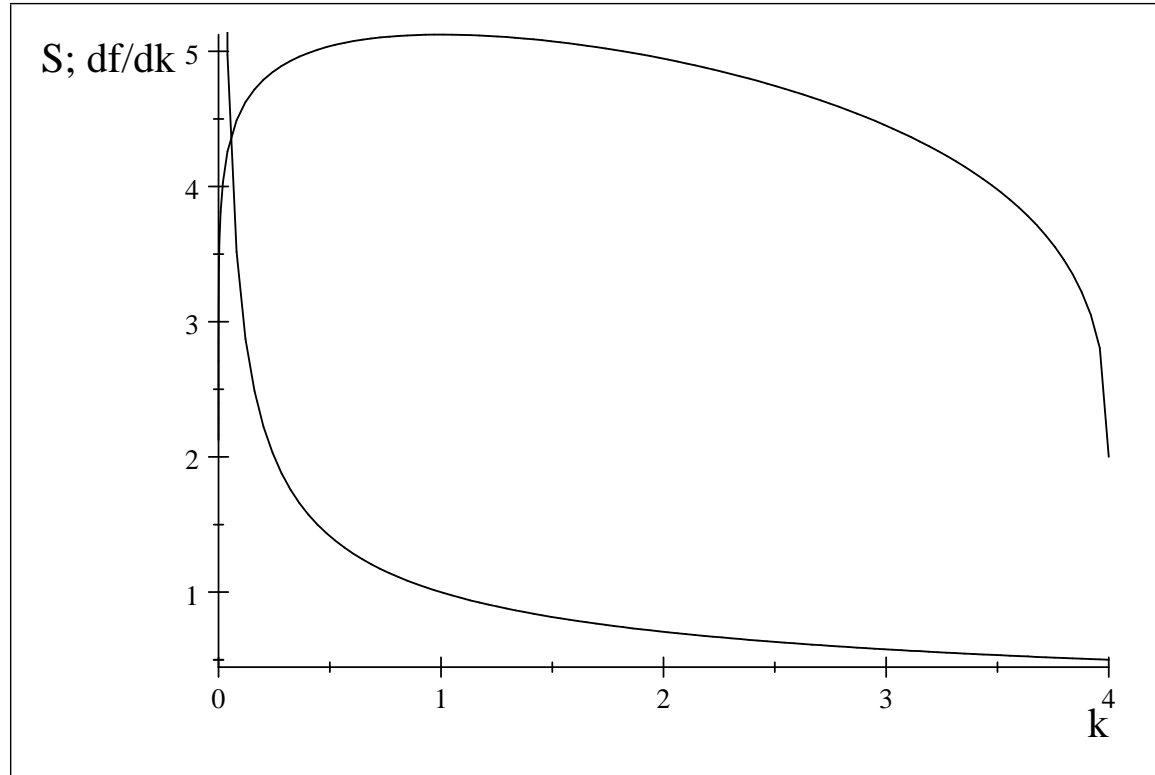

Figure 4: The Inverse Supply and Demand Functions. 


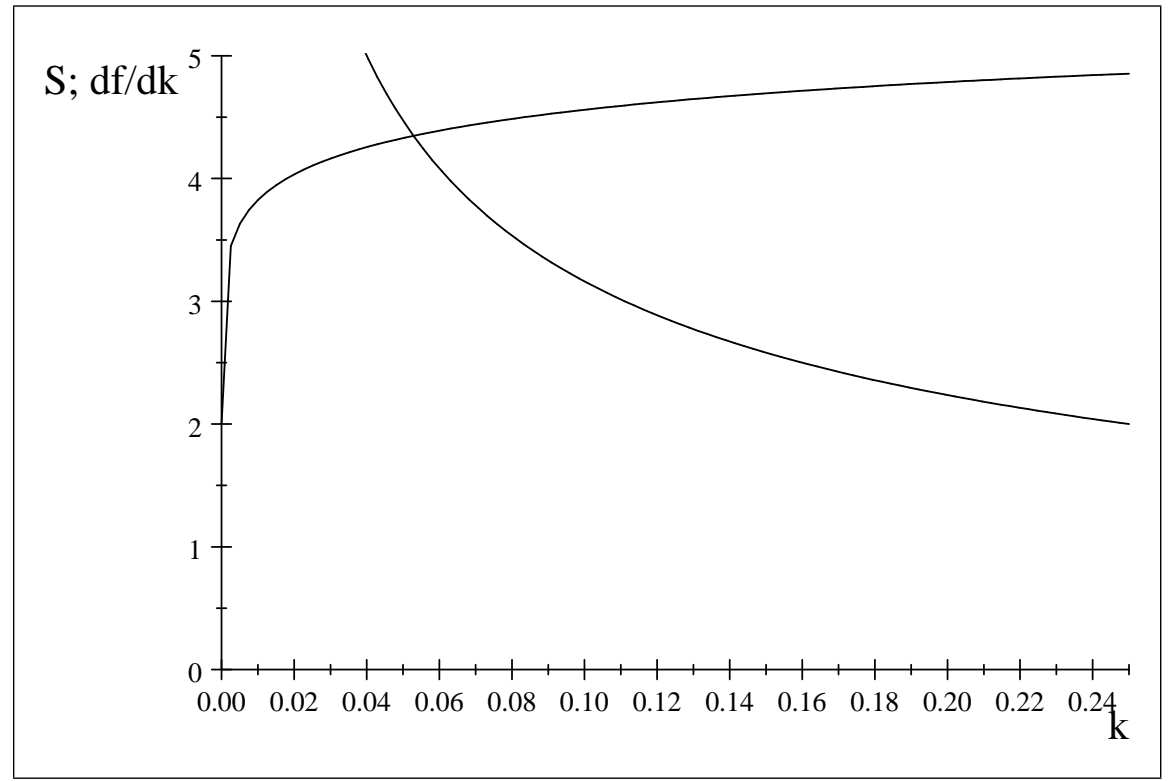

Figure 5: The Inverse Supply and Demand Functions. Zoomed View 


\section{References}

[1] Łukasz Balbus, "On Recursive Utilities for Non-Affine Aggregators and Conditional Certainty Equivalent," Economic Theory, published online, September 2019.

[2] Łukasz Balbus, Kevin Reffett, and Łukasz Woźny, "Time Consistent Markov Policies in Dynamic Economies with Quasi-Hyperbolic Consumers," International Journal of Game Theory, 44, 2015, pp. 83-112.

[3] Robert A. Becker and John H. Boyd III, Capital Theory, Equilibrium Analysis, and Recursive Utility, Blackwell Publishing, 1997.

[4] Robert A. Becker and Juan Pablo Rincón-Zapatero, "Recursive Utility and Thompson Aggregators,I: Constructive Existence Theory for the Koopmans Equation" Indiana University CAEPR Working Paper \#2018-006, July 16, 2018.

[5] Robert A. Becker and Juan Pablo Rincón-Zapatero, "Recursive Utility and Thompson Aggregators,II: Uniqueness of the Recursive Utility Representation" Indiana University CAEPR Working Paper \#2018-008, August $10,2018$.

[6] Richard Beals and Tjalling C. Koopmans, "Maximizing Stationary Utility in a Constant Technology," SIAM Journal on Applied Mathematics, Vol. 17(5), 1969, 1001-1015.

[7] Jess Benhabib, Mukul Majumdar, and Kazuo Nishimura, "Global Equilibrium Dynamics with Stationary Recursive Preferences," Journal of Economic Behavior and Organization, 8, 1987, pp. 429-452.

[8] Christopher Bliss, Capital Theory and the Distribution of Income, NorthHolland Publishing, 1975.

[9] Gaetano Bloise and Yiannis Vailakis, "Convex Dynamic Programming with (Bounded) Recursive Utility," Journal of Economic Theory, 173, 2018, pp. 118-141.

[10] John H. Boyd III, "Recursive Utility and the Ramsey Problem," Journal of Economic Theory, 50, 1990, pp. 326-345.

[11] Andrzej Granas and James Dugundji, Fixed Point Theory, Springer, New York, 2003.

[12] Leonid Kantorovich, "The Method of Successive Approximations for Functional Equations," Acta Mathematica, Vol. 71, 1939, 63-97.

[13] Tjalling C. Koopmans, "Stationary Ordinal Utility and Impatience," Econometrica, 28, 1960, pp. 287-309. 
[14] Tjalling C. Koopmans, "Objectives, Constraints, and Outcomes in Optimal Growth Theory," Econometrica, 35, 1967, pp. 1-15.

[15] Tjalling C. Koopmans, "Representation of Preference Orderings Over Time," in Decisions and Organization (C.B. McGuire and Roy Radner, eds.) North-Holland, Amsterdam, 1972 (Second Edition, 1986).

[16] Tjalling C. Koopmans, Peter A. Diamond, and Richard E. Williamson, "Stationary Utility and Time Perspective," Econometrica, 82, 1964, pp. $82-100$.

[17] Robert E. Lucas and Nancy L. Stokey, "Optimal Growth with Many Consumers," Journal of Economic Theory, 32, 1984, pp. 139-171.

[18] Michael J.P. Magill and Kazuo Nishimura, "Impatience and Accumulation," Journal of Mathematical Analysis and Applications, 98, 1984, 270281.

[19] Massimo Marinacci and Luigi Montrucchio, "Unique Solutions for Stochastic Recursive Utilities," Journal of Economic Theory, 145, 2010, pp. 17761804 .

[20] Massimo Marinacci and Luigi Montrucchio, "Unique Tarski Fixed Points," Mathematics of Operations Research, 2019 (online 04 June 2019).

[21] A. Tarski, "A Lattice-Theoretical Fixpoint Theorem and Applications," Pacific Journal of Mathamatics, 3, 1955, pp. 285-309. 